\title{
Retrograde Changes in Presynaptic Function Driven by Dendritic mTORC1
}

\author{
Fredrick E. Henry, ${ }^{1,2}$ Amber J. McCartney, ${ }^{1,2}$ Ryan Neely, ${ }^{2}$ Amanda S. Perez, ${ }^{2}$ Cynthia J. L. Carruthers, ${ }^{2}$ \\ Edward L. Stuenkel, ${ }^{1,4}$ Ken Inoki, ${ }^{3,4}$ and Michael A. Sutton ${ }^{1,2,4}$ \\ ${ }^{1}$ Neuroscience Graduate Program, ${ }^{2}$ Molecular and Behavioral Neuroscience Institute, ${ }^{3}$ Life Sciences Institute, and ${ }^{4}$ Department of Molecular and \\ Integrative Physiology, University of Michigan, Ann Arbor, Michigan 48109
}

\begin{abstract}
Mutations that alter signaling through the mammalian target of rapamycin complex 1 (mTORC1), a well established regulator of neuronal protein synthesis, have been linked to autism and cognitive dysfunction. Although previous studies have established a role for mTORC1 as necessary for enduring changes in postsynaptic function, here we demonstrate that dendritic mTORC1 activation in rat hippocampal neurons also drives a retrograde signaling mechanism promoting enhanced neurotransmitter release from apposed presynaptic terminals. This novel mode of synaptic regulation conferred by dendritic mTORC1 is locally implemented, requires downstream synthesis of brain-derived neurotrophic factor as a retrograde messenger, and is engaged in an activity-dependent fashion to support homeostatic trans-synaptic control of presynaptic function. Our findings thus reveal that mTORC1-dependent translation in dendrites subserves a unique mode of synaptic regulation, highlighting an alternative regulatory pathway that could contribute to the social and cognitive dysfunction that accompanies dysregulated mTORC1 signaling.
\end{abstract}

\section{Introduction}

Although principal neurons receive many thousands of synapses, the ability to tune synaptic strength independently along specific regions of the dendritic arbor is thought to confer the increased computational capacity necessary for new learning and memory storage in neural circuits (Rabinowitch and Segev, 2008; Branco and Haüsser, 2010). The discovery of polyribosomes in the distal dendrites of dentate granule neurons (Steward and Levy, 1982) first suggested that dendritic translation might participate in controlling synaptic function on a local level, an idea that has been clearly substantiated by work in a wide range of model systems (Kang and Schuman, 1996; Martin et al., 1997; Huber et al., 2000; Sutton et al., 2006). In particular, the mammalian target of rapamycin (mTOR) is known to be an important control point for protein synthesis related to synaptic plasticity (Casadio et al., 1999; Tang et al., 2002; Cammalleri et al., 2003; Gong et al., 2006) and memory (Tischmeyer et al., 2003; Dash et al., 2006; Blundell et al., 2008).

mTOR is a serine/threonine protein kinase that integrates into two distinct protein complexes, mTOR complex 1 (mTORC1)

Received May 3, 2012; revised Aug. 31, 2012; accepted 0ct. 1, 2012.

Author contributions: F.E.H. and M.A.S. designed research; F.E.H., A.J.M., R.N., A.S.P., and C.J.L.C. performed research; E.L.S. and K.I. contributed unpublished reagents/analytic tools; F.E.H., A.J.M., R.N., A.S.P., C.J.L.C., and M.A.S. analyzed data; F.E.H. and M.A.S. wrote the paper.

This work was supported by Grants F31MH093112 (F.E.H.) and R01MH085798 (M.A.S.) from The National Institute of Mental Health and a grant from the Pew Biomedical Scholars Program (M.A.S.). We thank Robert Edwards for generously providing vglut1-pHluorin. We also thank Hisashi Umemori and members of the Sutton laboratory for many helpful discussions.

Correspondence should be addressed to Michael A. Sutton, Molecular and Behavioral Neuroscience Institute, 5067 BSRB, 109 Zina Pitcher Place, University of Michigan, Ann Arbor, MI 48109-2200. E-mail: masutton@umich.edu.

DOI:10.1523/JNEUROSCI.2149-12.2012

Copyright $\odot 2012$ the authors $\quad 0270-6474 / 12 / 3217128-15 \$ 15.00 / 0$ and mTOR complex 2 (mTORC2) (Ma and Blenis, 2009). Of these, mTORC1 promotes translation of specific classes of $\mathrm{mR}$ NAs via phosphorylation of downstream effectors, such as eIF4E binding protein (4EBP) and p70 S6 kinase (S6K1) (Wang and Proud, 2006; Costa-Mattioli et al., 2009; Zoncu et al., 2011). A wide array of extracellular signals regulates mTORC1 signaling through the mTORC1 repressor tuberous sclerosis complex (TSC) or the small GTPase Rheb, which promotes mTORC1 activation (Yang and Guan, 2007).

Animal studies have clearly implicated mTORC1 in hippocampal synaptic plasticity and in the formation of long-term hippocampal-dependent memories (Ehninger et al., 2008; Gkogkas et al., 2010; Hoeffer and Klann, 2010). Moreover, human mutations that result in dysregulation of mTORC1 signaling have been associated with vulnerability to autism, cognitive impairment, and epilepsy (Onda et al., 2002; Butler et al., 2005; Johannessen et al., 2005; Kelleher and Bear, 2008), underscoring the importance of this signaling complex for proper neural circuit function in the human brain. At vertebrate synapses, dendritic mTORC1 has been most clearly implicated in enduring forms of long-term potentiation (LTP) and long-term depression (LTD) that are induced and expressed postsynaptically (Tang et al., 2002; Cammalleri et al., 2003, Sharma et al., 2010; Hoeffer et al., 2008), suggesting that mTORC1-dependent translational control may participate in maintaining altered AMPA receptor (AMPAR) expression at synapses, promoting structural synaptic changes, or both.

Here, we demonstrate that mTORC1-dependent translation in dendrites exerts a novel form of regulatory control over synapse function. Unlike enduring changes in postsynaptic function during LTP and LTD, we find that homeostatic enhancement of postsynaptic function induced by acute loss of excitatory synaptic 
drive occurs via mTORC1-independent protein synthesis. Instead, we find that dendritic mTORC1 activation is both necessary and sufficient for driving protein synthesis-dependent compensatory changes in neurotransmitter release from apposed presynaptic terminals, revealing that parallel translational control pathways operate locally in dendrites to mediate distinct homeostatic adaptations at synapses.

\section{Materials and Methods}

Cell culture and electrophysiology. Dissociated postnatal hippocampal neuron cultures, prepared from postnatal day 1-2 rat pups of either sex, were plated at a density of $230-460 \mathrm{~mm}^{2}$ in poly-D-lysine-coated glassbottom Petri dishes (Mattek), as previously described (Jakawich et al., 2010). Cultures were maintained for at least $21 \mathrm{DIV}$ at $37^{\circ} \mathrm{C}$ in growth medium [Neurobasal A supplemented with B27 and Glutamax-1 (Invitrogen)] before use. Whole-cell patch-clamp recordings were made with an Axopatch 200B amplifier from cultured hippocampal neurons bathed in HEPES-buffered saline (HBS) containing (in mM) the following: 119 $\mathrm{NaCl}, 5 \mathrm{KCl}, 2 \mathrm{CaCl}_{2}, 2 \mathrm{MgCl}_{2}$, 30 Glucose, 10 HEPES, pH 7.4. Wholecell pipette had resistances ranging from 3 to $6 \mathrm{M} \Omega$. Internal solution contained (in mM) the following: 100 cesium gluconate, 0.2 EGTA, 5 $\mathrm{MgCl}_{2}, 2$ adenosine triphosphate, 0.3 guanosine triphosphate, 40 HEPES, $\mathrm{pH}$ 7.2. mEPSCs were recorded at $-70 \mathrm{mV}$ from neurons with a pyramidal-like morphology in the presence of $1 \mu \mathrm{M}$ TTX and $10 \mu \mathrm{M}$ bicuculline and analyzed off-line using Synaptosoft minianalysis software. sEPSCs in active networks were recorded at $-70 \mathrm{mV}$ in modified HBS (as above, except with $0.5 \mathrm{~mm} \mathrm{CaCl}$ and $3.5 \mathrm{~mm} \mathrm{MgCl}_{2}$ ) without TTX or bicuculline. Total excitatory synaptic input onto recorded neurons was assessed by computing charge transfer $>30$ randomly selected $1 \mathrm{~s}$ intervals for each recording. NMDAR EPSCS were recorded in HBS with $0 \mathrm{MgCl}_{2}$ and were evoked with a bipolar stimulating electrode positioned close to the recorded neuron. Once a stable response was obtained to stimuli delivered at $0.33 \mathrm{~Hz}, \mathrm{MK} 801(20 \mu \mathrm{M})$ was applied to the bath for 5 min without stimulation. Following wash in, 200 stimulations were delivered in the presence of MK801. The peak of the NMDAR current was measured for each and normalized to the first response. Group comparisons are presented as the normalized mean peak NMDAR current as a function of number of stimulations in the presence of MK801. Statistical differences between experimental conditions were determined by ANOVA and post hoc Fisher's least significant difference (LSD) test, unless otherwise indicated.

$\mathrm{Ca}^{2+}$ phosphate transfection. Plasmids encoding TSC1, TSC2, RhebWT, and RhebQ64L were used as previously described (Inoki et al., 2002, 2003). vglut-pHluorin was generously provided by Robert Edwards. To achieve sparse expression, neurons were transfected with 0.5 $\mu \mathrm{g}$ of total DNA using the $\mathrm{Ca}^{2+}$ phosphate CalPhos Transfection kit (ClonTech) according to the manufacturer's protocol. Unless otherwise indicated, all experiments were performed $24 \mathrm{~h}$ post-transfection.

Immunocytochemisty and microscopy. Except for experiments involving vglut-pHluorin, all imaging was performed on an inverted Olympus FV1000 laser-scanning confocal microscope using a Plan-Apochromat $63 \times / 1.4$ oil objective with $1 \times$ or $2 \times$ digital zoom. Alexa 488 was excited with the $488 \mathrm{~nm}$ line of an argon ion laser and emitted light was typically collected between 500 and $530 \mathrm{~nm}$ with a tunable emission filter. Alexa $555 / 568$ were excited with a $559 \mathrm{~nm}$ diode laser and emitted light was typically collected between 570 and $670 \mathrm{~nm}$. Before image collection, the acquisition parameters for each channel were optimized to ensure a dynamic signal range and to ensure no signal bleed-through between detection channels. For every experiment, identical parameters were used for each treatment option. We verified that no detectable staining was observed in control samples incubated in the absence of primary antibody (but otherwise processed identically).

For BDNF and phospho-S6 (PS6) staining, cells were treated in conditioned media as indicated, then fixed at room temperature for $20 \mathrm{~min}$ with $4 \%$ paraformaldehyde (PFA)/4\% sucrose in PBS with $1 \mathrm{~mm} \mathrm{MgCl}_{2}$ and $0.1 \mathrm{~mm} \mathrm{CaCl}_{2}$ (PBS-MC). After washing in PBS-MC (5 min), cells were permeabilized ( $0.1 \%$ Triton X in PBS-MC, 5 min), blocked with $2 \%$ bovine serum albumin in PBS-MC for $30 \mathrm{~min}$, and labeled with a rabbit polyclonal antibody against BDNF (Santa Cruz Biotechnology, 1:100), or a rabbit polyclonal S235/236 phosphospecific antibody (Cell Signaling Technology; 1:50); for each, colabeling and a mouse monoclonal antibody against MAP2 (Sigma-Aldrich, 1:5000) for either $60 \mathrm{~min}$ at room temperature or overnight at $4^{\circ} \mathrm{C}$. Alexa 555-conjugated goat anti-rabbit (1:500) and Alexa 635-conjugated goat anti-mouse (1:500) secondary antibodies (each $60 \mathrm{~min}$ at room temperature) were used to visualize BDNF/PS6 and MAP2 staining, respectively. To ensure blind sampling of BDNF/PS6 expression, neurons with a pyramidal-like morphology were selected for imaging by epifluorescent visualization of MAP2 staining. Analysis of expression in somatic or dendritic neuronal compartments was performed on maximal intensity $z$-compressed image stacks. Dendrites were linearized and extracted from the full-frame image using the straighten plug-in for ImageJ. For both somatic and dendritic signal, expression was estimated by the average nonzero pixel intensity for each compartment. To analyze dendritic BDNF expression, dendrites were linearized and extracted from the full-frame image using the straighten plug-in for ImageJ. To combine data across multiple experimental runs of the same experiment, expression in each image was normalized against the average nonzero pixel intensity for the respective control group. In experiments where BDNF or PS6 were assessed in transfected cells, neurons were also colabeled with a chicken polyclonal antibody against GFP (Millipore, 1:1000) and an Alexa 488-conjugated goat anti-chicken secondary antibody. Statistical differences were assessed by ANOVA, followed by Fisher's LSD post hoc tests.

For analysis of surface GluA1 expression, neurons were live-labeled with a rabbit polyclonal antibody recognizing a surface epitope of GluA1 ( $10 \mu \mathrm{g} / \mathrm{ml}$, EMD Biosciences) for $15 \mathrm{~min}$ at $37^{\circ} \mathrm{C}$, followed by fixation (2\% PFA in PBS-MC for 15 min at room temperature), and immunocytochemical labeling with a mouse monoclonal anti-PSD95 antibody (1: 1000, Millipore Bioscience Research Reagents) as described above. Alexa 488-conjugated goat anti-mouse (1:500) and Alexa 555-conjugated goat anti-rabbit (1:500) secondary antibodies (each $60 \mathrm{~min}$ at room temperature) were used to visualize PSD95 and GluA1 staining, respectively. Neurons were selected based on PSD95 epiflourescence to ensure blind sampling of surface GluA1. For analysis, "synaptic" GluR1 was defined as a particle that occupied $>10 \%$ of the area defined by a PSD95 particle, and the average integrated intensity (total number of nonzero pixels times intensity) of synaptic GluR1 particles was calculated using custom written analysis routines for ImageJ. Analysis was performed on both full-frame images as well on dendrites straightened and extracted from the full-frame image, where " $n$ " equals the number of images or number of dendrites, respectively. Both analysis methods yielded similar results. Statistical differences were assessed by ANOVA, followed by Fisher's LSD post hoc tests.

Optical analysis of presynaptic function used live-labeling with an Oyster 550-conjugated rabbit polyclonal antibody against the lumenal domain of synaptotagmin 1 (syt-lum; 1:100, Synaptic Systems). Before labeling, neurons were treated with $2 \mu \mathrm{M}$ TTX for $15 \mathrm{~min}$ to isolate spontaneous neurotransmitter release. Neurons were then labeled with anti-syt-lum for $5 \mathrm{~min}$ at room temperature, washed, fixed with $4 \%$ $\mathrm{PFA} / 4 \%$ sucrose in PBS-MC, permeabilized, and blocked as above, then labeled with a guinea pig polyclonal anti-vglut 1 antibody (1:2500, Millipore Bioscience Research Reagents). The intrinsic fluorescent signal of the anti-syt-lum at synaptic sites was amplified by an Alexa 555conjugated goat anti-rabbit (1:500) secondary antibody. An Alexa 488conjugated goat anti-guinea pig (1:250) secondary antibody (each for 60 $\mathrm{min}$ at room temperature) was used to visualize vglut1 staining. Neurons were selected based on vglut 1 epiflourescence to ensure blind sampling of syt-lum expression. For analysis, a "synaptic" syt-lum particle was defined as a particle that occupied $>10 \%$ of the area defined by a vglut 1 particle, and the proportion of vglut 1 particles containing synaptic sytlum particles was calculated using custom written analysis routines for ImageJ. Statistical differences were assessed by ANOVA, followed by Fisher's LSD post hoc tests.

Local perfusion. All local perfusion experiments were performed on an inverted Olympus FV1000 laser-scanning confocal microscope using Plan-Apochromat 40×/0.95 air (for live imaging) and Plan-Apochromat $40 \times / 1.0$ oil (for retrospective imaging) objectives. Stable microperfusion 
was achieved by use of a dual micropipette delivery system, as described previously (Sutton et al., 2006). The delivery micropipette was pulled as a typical whole-cell recording pipette with an aperture of $\sim 0.5 \mu \mathrm{m}$. The area of dendrite targeted for local perfusion was controlled by a suction pipette, which was used to draw the treatment solution across one or more dendrites and to remove the perfusate from the bath. A cellimpermeant fluorescent dye (Alexa $488,1 \mu \mathrm{g} / \mathrm{ml}$ ) was included in the perfusate to visualize the affected area. In all local perfusion experiments, the bath was maintained at $37^{\circ} \mathrm{C}$ and continuously perfused at $1.5 \mathrm{ml} /$ min with HBS.

For analysis, the size of the treated area was determined in each linearized dendrite based on Alexa 488 fluorescence integrated across all images taken during local perfusion. Adjacent nonoverlapping dendritic segments, $25 \mu \mathrm{m}$ in length, proximal (i.e., toward the cell soma) and distal to the treated area were assigned negative and positive values, respectively. To examine localized changes in syt-lum uptake, $2 \mu \mathrm{M}$ TTX was bath applied for $10 \mathrm{~min}$ to isolate spontaneous neurotransmitter release immediately following local perfusion. Neurons were then livelabeled with syt-lum for $5 \mathrm{~min}$ at room temperature, and processed for immunocytochemistry as described above. To assess relative changes in syt-lum and vglut1 staining in perfused dendrites, the average nonzero pixel intensity was calculated for each dendritic segment. The average value in untreated segments was assigned a value of 1 , which was then used to normalize vlgut and syt-lum intensity in all segments (including the treated area). Statistical differences in these measurements between syt and vglut at each segment were assessed by Student's $t$ test.

vglut1-pHluorin imaging. Cultured hippocampal neurons were transfected at DIV 14-16 with vglut-pHluorin as well as mCherrysynaptophysin to aid in visual identification of terminal boutons. Experiments were conducted 2-3 d post-transfection in HBS containing $10 \mu \mathrm{M}$ CNQX and $50 \mu \mathrm{M}$ DL-APV. Action potentials were evoked via a Grass stimulator unit using a custom fabricated electric field stimulator, which was inserted into each dish just before start of an experiment. Each stimulus consisted of a $2 \mathrm{~ms} 10 \mathrm{~V}$ potential difference between platinum-iridium wires $6 \mathrm{~mm}$ apart. To observe changes in evoked release, we recorded the change in vglut-pHluorin fluorescence intensity elicited by delivering stimulus repetitions at $10 \mathrm{~Hz}$ for $10 \mathrm{~s}$, which has been shown to provide a measure of exocytosis undiluted by signal degradation due to endocytosis or reacidification (Kim and Ryan, 2009). Each stimulus train was delivered three times for each set of synapses, with a 5 min interval separating each train. Images were taken using a Hammamatsu EM-CCD camera mounted on an inverted microscope (Olympus IX8) using a $40 \times 1.3$ NA oil-immersion objective (Olympus). The filter set consisted of a 472/30 excitation filter, a 495 edge dichroic mirror, and a 520/30 emission filter. Illumination was achieved via a Sutter Lambda LS/OR30 $300 \mathrm{~W}$ xenon bulb control by a Sutter Lambda 10 shutter (Sutter Instrument). All images were captured at a depth of 16 bits to a Dell Optiplex computer using MetaMorph software (Molecular Devices). Full frame $(512 \times 512,16 \mu \mathrm{m}$ pixels $)$ imaging sequences were acquired at a rate of $5 \mathrm{~Hz}$ using $50 \mathrm{~ms}$ exposure for vglut-pHluorin. Image sequences were analyzed using the "time series" plug-in for ImageJ (http://rsb.info.nih.gov/ij/plugins/time-series.html). Active synapses were identified for each experiment by subtracting the signal before the initiation of each stimulus train from the peak increase in vglut-pHluorin signal elicited by the train. Rectangular ROIs $(1.55 \times$ $1.55 \mu \mathrm{m}$ ) were placed over each of the resulting puncta in the "difference image." The time-lapse plug-in was then used to measure average fluorescence for each ROI over time. To correct for photobleaching during image series acquisition, an identically sized ROI was positioned in a nonsynaptic region adjacent to an identified release site. Measurements obtained from this nonsynaptic ROI were fit with a biexponential decay function, and these values were then subtracted from the data traces associated with active release sites. Fluorescence signals were quantified as $\Delta F / F 0$, where $F 0$ is defined as the average of five sequential images recorded before the onset of stimulation for each ROI.

Western blotting. Samples were collected in lysis buffer containing 100 mм NaCl, 10 mм NaPO$, 10 \mathrm{~mm} \mathrm{Na}_{4} \mathrm{P}_{2} \mathrm{O}_{7}, 10$ mm lysine, 5 mм EDTA, 5 mm EGTA, $50 \mathrm{~mm} \mathrm{NaF,} 1 \mathrm{~mm} \mathrm{NaVO}$, 1\% Triton-X, 0.1\% SDS, and 1 tablet Complete Mini protease inhibitor mixture (Roche)/7 ml, pH 7.4.
Equal amounts of protein for each sample were loaded and separated on $12 \%$ polyacrylamide gels, then transferred to PVDF membranes. Blots were blocked with Tris-buffered saline containing $0.1 \%$ Triton-X (TBST) and 5\% nonfat milk for $60 \mathrm{~min}$ at room temperature, and incubated with a rabbit polyclonal primary antibody against BDNF (Santa Cruz Biotechnology, 1: 200) for either $60 \mathrm{~min}$ at room temperature or overnight at $4^{\circ} \mathrm{C}$. After washing with TBST, blots were incubated with HRP-conjugated anti-rabbit or anti-mouse secondary antibody (1:5000; Jackson Immunoresearch), followed by enhanced chemiluminescent detection (GE Healthcare). All blots were probed with a mouse monoclonal antibody against $\alpha$-tubulin (1:5000, Sigma-Aldrich) to confirm equal loading. Band intensity was quantified with densitometry with NIH ImageJ and expressed relative to the matched control sample. Statistical differences between treatment conditions and control were assessed first by an overall nonparametric ANOVA (Kruskal-Wallis test) followed by a post hoc Wilcoxon signed rank test for individual comparisons.

\section{Results}

\section{Loss of excitatory synaptic input drives dendritic mTORC1 activation}

Previous work has established that strong synaptic activation can drive mTORC1 signaling in hippocampal neurons (Tang et al., 2002; Cammalleri et al., 2003), but it is largely unclear how the activity-dependent regulation of mTORC1 signaling is sculpted by loss of synaptic drive. To explore this question, we examined the regulation of several downstream targets of mTORC1 signaling in active networks of cultured hippocampal neurons subject to acute blockade of excitatory transmission through either AMPARs or NMDARs. Treating cultured hippocampal neurons with the AMPAR antagonist CNQX $(40 \mu \mathrm{M})$, but not the NMDAR antagonist APV $(50 \mu \mathrm{M})$, induced a rapid time-dependent increase in phosphorylation of ribosomal protein S6 at S235/236 (Fig. 1AC), a downstream target of mTORC1 activation. Likewise, CNQX treatment $(40 \mu \mathrm{M}, 30 \mathrm{~min})$ induced phosphorylation of S6K1 at Thr389 and 4EBP at Thr37/46 (Fig. 1D,E), both direct mTORC1 phosphorylation sites. The mTORC1 inhibitor rapamycin (100 nM) completely suppressed both basal and enhanced S6 and S6K1 phosphorylation (Fig. 1C,D), as well as enhanced phosphorylation of 4EBP1 (Fig. $1 E$ ), confirming a role for $\mathrm{mTORC1}$ activation in these effects. Another AMPAR antagonist, NBQX $(10 \mu \mathrm{M})$, produced similar rapamycin-sensitive effects on S6, S6K1, and 4EBP1 phosphorylation (data not shown). Finally, immunocytochemistry revealed that AMPAR blockade induced significant S6 phosphorylation in both the cell bodies and dendrites of hippocampal neurons (Fig. $1 F, G)$, whereas MAP2 expression from these same neurons was unchanged. Under our staining conditions, virtually all PS6 immunofluorescence was abolished by pretreatment with $100 \mathrm{~nm}$ rapamycin (data not shown), verifying PS6 staining as a valid readout of mTORC1 activation. These results indicate that mTORC1 signaling is activated in response to acute loss of excitatory synaptic drive as well as in response to patterned synaptic activation.

A drop in excitatory synaptic input is also known to regulate translation through dephosphorylation of eukaryotic elongation factor 2 (eEF2) at Thr56 (Sutton et al., 2007; Nosyreva and Kavalali, 2010). eEF2 kinase can be regulated by mTORC1 activation (Wang and Proud, 2006), so we also examined eEF2 phosphorylation in response to AMPAR blockade and its reliance on intact mTORC1 signaling. While AMPAR blockade induced rapid eEF2 dephosphorylation consistent with translational activation, this effect was insensitive to coincident mTORC1 inhibition with rapamycin (Fig. $1 \mathrm{H}, \mathrm{I}$ ). These results suggest that AMPAR blockade engages multiple translational regulatory pathways in hippocampal neurons. 

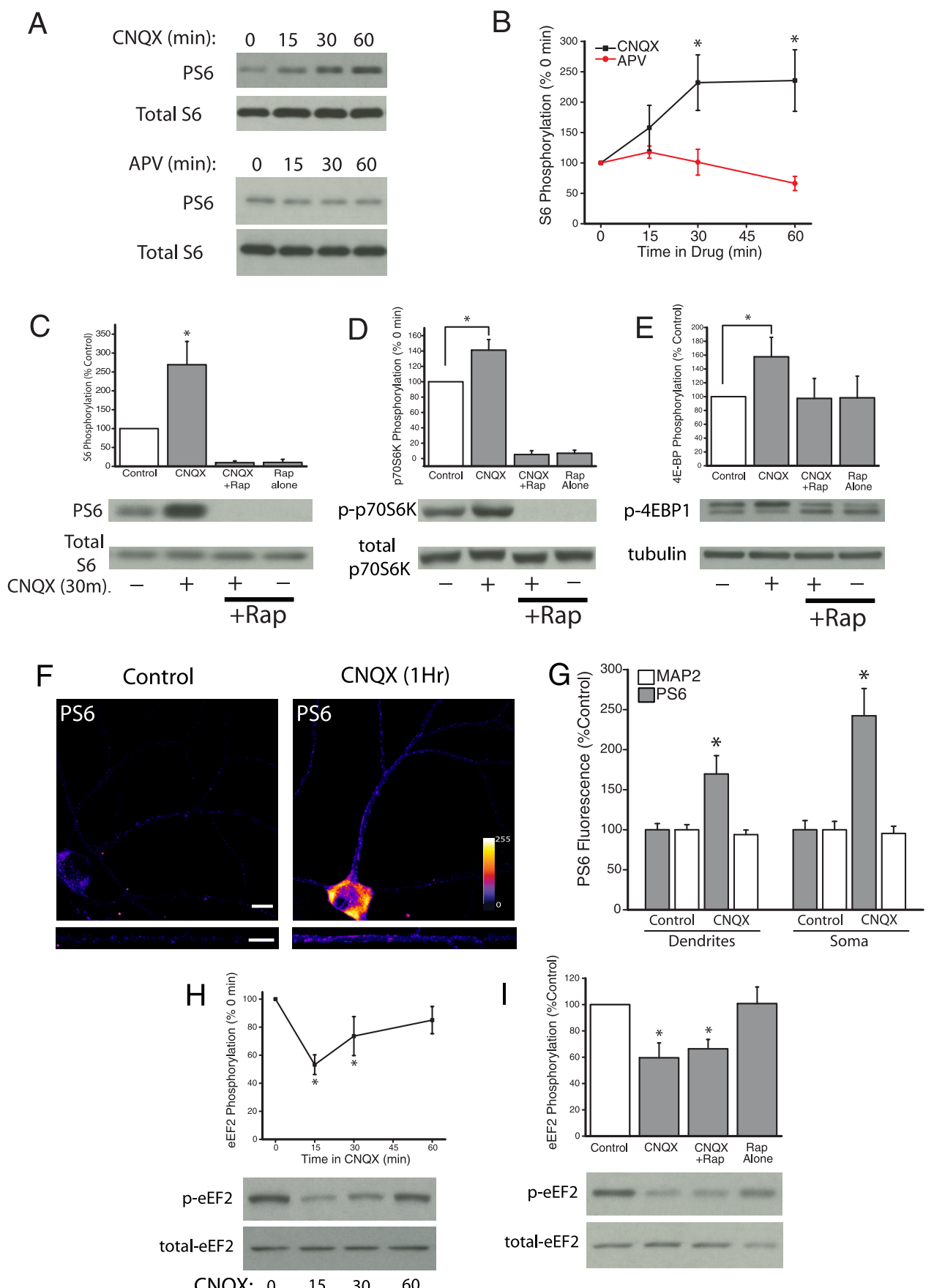

Figure 1. Acute loss of excitatory synaptic drive activates dendriticmTORC1 signaling.A, Representative Western blots depicting phosphorylated (S235/236) and total ribosomal protein S6 following AMPAR blockade with $40 \mu \mathrm{M}$ CNQX ( $n=8$ experiments) or NMDAR blockade with $50 \mu \mathrm{M}$ APV ( $n=6$ experiments) for the indicated times. B, Mean ( \pm SEM) expression of PS6 in neurons subject to AMPAR or NMDAR blockade; AMPAR blockade induced a significant $\left({ }^{*} p<0.05\right.$ relative to 0 min control) increase in 56 phosphorylation, whereas NMDAR blockade did not. $(-E$, Representative Western blots and summary data from experiments $(n=5)$ where neurons were treated with CNQX ( $40 \mu \mathrm{m}, 30 \mathrm{~min})$ plus rapamycin ( $100 \mathrm{~nm}, 30$ min pretreatment) before harvesting; ${ }^{*} p<0.05$ relative to controls. AMPAR blockade stimulates phosphorylation of downstream mTORC1 effectors ribosomal protein S6 (C), p70S6K (D), and 4E-BP1 (E) (upper band) in a rapamycin-sensitive fashion. The increase in phosphorylation of 56 , p70S6K, as well as 4E-BP1 accompanying AMPAR blockade is prevented by rapamycin ( ${ }^{*} p<0.05$ relative to untreated control). $\boldsymbol{F}$, Representative examples of PS 6 staining from cells treated with CNQX ( $40 \mu \mathrm{M}, 60 \mathrm{~min}$ ) or control; straightened dendrites are shown below each full-frame image. Scale bar, $10 \mu \mathrm{m}$. PS6 fluorescence intensity indicated by color look-up table. G, Mean (+SEM) expression of MAP2 and PS6 in somatic and dendritic compartments, normalized to average control values, in neurons treated as indicated. PS6 fluorescence was significantly ( ${ }^{*} p<0.05$, relative to control) enhanced by AMPAR blockade ( $n=28$ cells) relative to control neurons $(n=30$ cells), in both the dendritic and somatic compartments. MAP2 expression did not differ between groups. $\boldsymbol{H}, \boldsymbol{I}$, Representative Western blots and summary data from experiments ( $n=8$ and 5 experiments for $\boldsymbol{H}$ and $\boldsymbol{I}$, respectively) assessing changes in eEF2 phosphorylation as a result of AMPAR blockade. Neurons were treated with CNQX plus rapamycin as above before harvesting; ${ }^{*} p<0.05$ relative to controls. AMPAR blockade elicits dephosphorylation of eEF2 (favoring translation elongation) that is insensitive to rapamycin and is characterized by kinetics distinct from mTORC1 activation.

Homeostatic enhancement of postsynaptic function is not dependent mTORC1 activation

We next examined whether activation of mTORC1 accompanying AMPAR blockade contributes to the homeostatic enhancement of postsynaptic function induced by such loss of synaptic activity, which is dependent on new protein synthesis (Sutton et al., 2006; Aoto et al., 2008). Indeed, rapid postsynaptic compensation shares with translation-dependent late-phase LTP (L-LTP) in the hip- 
pocampus a recruitment of AMPARs to synapses (Makino and Malinow, 2009), and mTORC1 signaling is known to be necessary for the enduring changes in postsynaptic function associated with L-LTP (Tang et al., 2002). Despite these similarities, we found that the increase in surface GluAl expression at synapses following AMPAR blockade was not altered by the mTORC1 inhibitor rapamycin (100 nM; Fig. 2A,B). Likewise, mTORC1 inhibition with either rapamycin or an $\mathrm{mTOR}$ active site inhibitor C-401 (10 $\mu \mathrm{M}$; Ballou et al., 2007) did not affect the rapid compensatory increase in amplitude of mEPSCs accompanying AMPAR blockade (40 $\mu \mathrm{M}$ CNQX, $3 \mathrm{~h}$ ), recorded immediately following CNQX washout (Fig. 2C,D). Similar to previous reports (Thiagarajan et al., 2005; Sutton et al., 2006; Aoto et al., 2008), we found that the homeostatic increase in mEPSC amplitude is mediated by recruitment of GluA1 homomeric receptors to synapses, which, unlike GluA2-containing AMPARs, are blocked by polyamine toxins, such as 1-napthylacetylspermine (NASPM). We found that mTORC1 inhibition coincident with AMPAR blockade also did not affect the NASPM sensitivity of scaled mEPSCs (Fig. $2 F, G)$, indicating that GluA1 homomeric receptors are recruited to synapses in the absence of $\mathrm{mTORC1}$ signaling. The effects of NASPM in these conditions are specific for mEPSC amplitude. While coincident rapamycin and C-401 treatment each block the increase in mEPSC frequency induced by AMPAR blockade, NASPM treatment after these treatments does not further reduce mEPSC frequency below control levels (data not shown). Together, these observations suggest that, while mTORC1 activation during L-LTP and mGluR-LTD is necessary for enduring changes in postsynaptic function (Hou and Klann, 2004; Antion et al., 2008), the homeostatic enhancement of postsynaptic function driven by loss of synaptic activity does not require mTORC1 signaling.

\section{mTORC1 is necessary for retrograde} enhancement of presynaptic function In addition to triggering rapid postsynaptic compensation, AMPAR blockade also drives compensatory increases in presynaptic function (Murthy et al., 2001; Thiagarajan et al., 2005) that depend on retrograde signaling from the postsynaptic cell (Jakawich et al., 2010; Lindskog et al., 2010; Groth et al., 2011). Thus, AMPAR blockade drives not only an increase in mEPSC amplitude, but also an increase in mEPSC frequency in hippocampal neurons. This increase in frequency reflects enhanced release probability from existing presynaptic terminals (Jakawich et al., 2010). Likewise, at the Drosophila neuromuscular junction, acute blockade of postsyn-
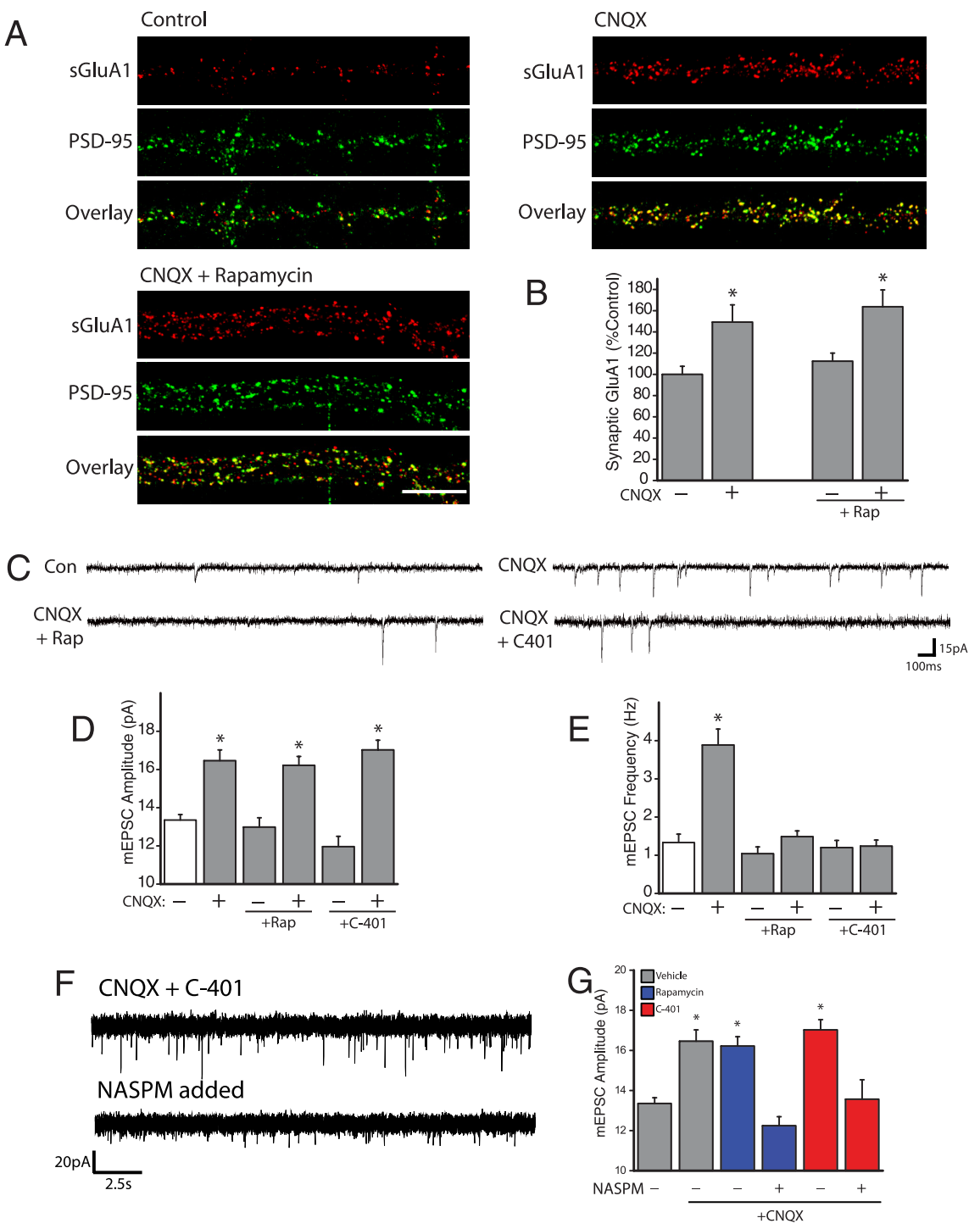

Figure 2. mTORC1 is not involved in adaptive postsynaptic compensation induced by AMPAR blockade. $\boldsymbol{A}, \boldsymbol{B}$, Representative examples ( $\boldsymbol{A}$ ) and mean (+SEM) normalized synaptic surface GluA1 (sGluA1) expression ( $\boldsymbol{B}$ ) in cultured neurons treated with 40 $\mu \mathrm{M}$ CNQX for $120 \mathrm{~min}$ or control ( $n=30$ images), with or without pretreatment with rapamycin (100 nм, 30 min before CNQX) ( $n=30 \mathrm{images} /$ group). mTORC1 activity is not required for enhanced synaptic sGluA1 expression after AMPAR blockade. Scale bar: $\boldsymbol{A}, 10 \mu \mathrm{m} .{ }^{*} p<0.05$ versus untreated control or rapamycin alone, respectively. $\boldsymbol{C}-\boldsymbol{E}$, Representative mEPSC recordings $(\boldsymbol{C})$ and mean (+SEM) mEPSC amplitude $(\boldsymbol{D})$ and frequency $(\boldsymbol{E})$ in neurons following washout of treatment with CNQX ( $40 \mu \mathrm{M}, 3 \mathrm{~h}$ ) with or without 30 min pretreatment with rapamycin $(100 \mathrm{~nm})$ or the mTOR active site inhibitor $\mathrm{C}-401(10 \mu \mathrm{M})$. Cell numbers for the indicated groups: control, $n=18 ; \mathrm{CNQX}, n=19 ;$ rapamycin alone, $n=7$; CNQX plus rapamycin, $n=13 ;$ C 401 alone, $n=11$; CNQX plus ( $401, n=10$. A significant $\left({ }^{*} p<0.05\right.$ vs control) increase in mEPSC amplitude and frequency follows AMPAR blockade; treatment with either mTORC1 inhibitor blocked the increase in mEPSC frequency but not the increase in mEPSC amplitude. $\boldsymbol{F}$, Representative recording from an example subjected to AMPAR blockade in the presence of $\mathrm{C}-401$, both before and after acute treatment with the polyamine toxin NASPM. G, Mean ( +SEM) mEPSC amplitude in neurons pretreated with either rapamycin (100 nM) or $(401(10 \mu \mathrm{M})$ and then subject to brief AMPAR blockade (CNQX, $40 \mu \mathrm{m}, 3 \mathrm{~h}$ ), followed by recording in the presence or absence of $10 \mu \mathrm{m}$ NASPM. Sample sizes (\# cells) for the indicated groups: control, $n=18 ; \mathrm{CNQX}, n=19 ;$ rapamycin alone, $n=7$; CNQX plus rapamycin, $n=13$; CNQX plus rapamycin plus NASPM, $n=12$; C401 alone, $n=11$; CNQX plus C401, $n=10$; CNQX plus C401 plus NASPM, $n=5$. Coincident mTORC1 inhibition did not affect the sensitivity of scaled mEPSCs to NASPM, indicating that the protein synthesis-dependent recruitment of GluA 1 homomeric receptors to synapses is established in an mTORC1-independent manner. ${ }^{*} p<0.05$ relative to untreated controls.

aptic AMPARs triggers rapid compensatory increases in quantal content (Frank et al., 2006), suggesting an evolutionarily conserved mode of regulation whereby postsynaptic glutamate receptors exert homeostatic control over presynaptic function. Interestingly, while mTORC1 activation is not necessary for compensatory enhancement of postsynaptic function following 

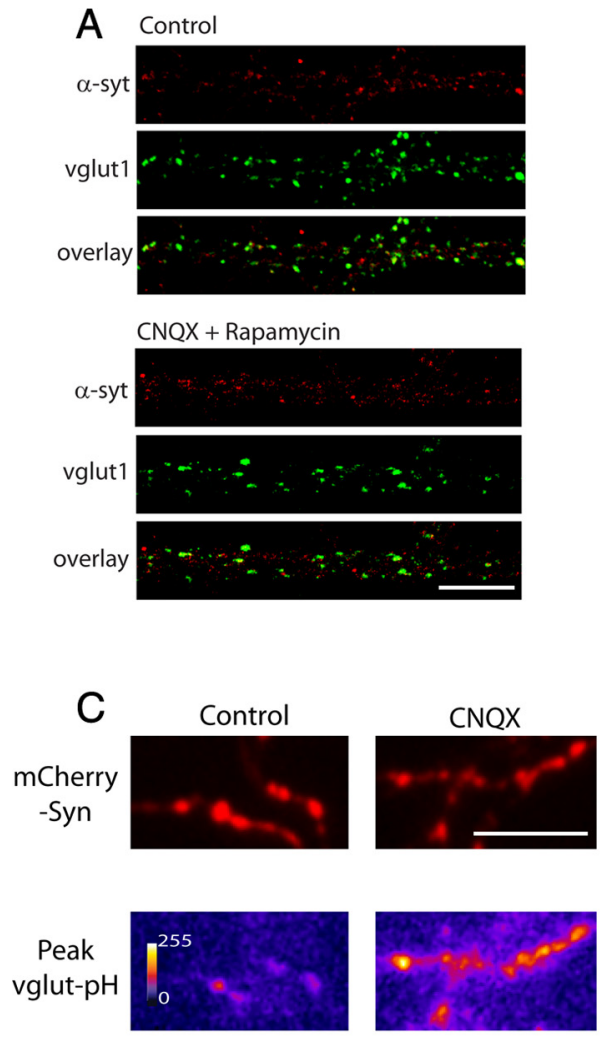
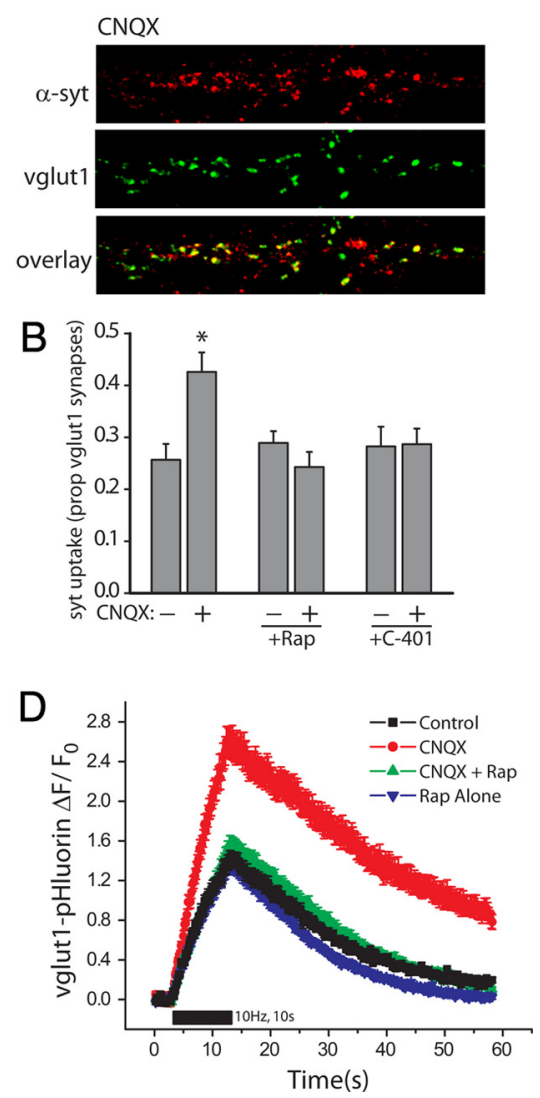

Figure 3. AMPAR blockade drives mTORC1-dependent enhancements in spontaneous and evoked vesicle release. $\boldsymbol{A}, \boldsymbol{B}, \mathrm{Repre}-$ sentative examples $(\boldsymbol{A})$ and mean (+SEM) syt-lum uptake $(\boldsymbol{B})$ from experiments where the indicated groups were pretreated (30 min before CNQX) with either rapamycin (100 nm) or C401 (10 $\mu \mathrm{m})$ and then subject to brief AMPAR blockade (CNQX, $40 \mu \mathrm{M}, 3 \mathrm{~h})$ before staining. Results for indicated groups: control, $n=30$ images; CNQX alone, $n=30$ images; rapamycin alone, $n=30$ images; CNQX plus rapamycin, $n=30$ images; (401 alone, $n=30$ images; CNQX plus C401, $n=30$ images. AMPAR blockade induces a significant $\left({ }^{*} p<0.05\right.$, relative to control) increase in syt-lum uptake prevented by coincident inhibition of mTORC1 activity. Scale bar: $\boldsymbol{A}, 10 \mu \mathrm{m}$. $\boldsymbol{C}, \boldsymbol{D}$, Example images of axon terminals coexpressing mCherry-synaptophysin (mCh-Syn, top) as well as vglut-pHluorin (vglut-pH, bottom). Bottom, Images of the same set of synapses after termination of a $10 \mathrm{~Hz} 10$ s stimulus train. Treatment with the AMPAR blocker CNQX ( $40 \mu \mathrm{m}, 3 \mathrm{~h}$ ) enhances the changes in vglut-pHluorin fluorescence intensity, compared with cells treated with vehicle in response to an identical stimulus train. Scale bar: $\boldsymbol{C}, 10 \mu \mathrm{m}$. D, Relative change in vglut-pHluorin fluorescence over time in response to a $10 \mathrm{~Hz} 10$ s stimulus train, recorded from cells treated with vehicle, CNQX, CNQX plus rapamycin, or rapamycin alone. Black bar represents onset of $10 \mathrm{~s}$ stimulus train. Black, Vehicle control ( $n=579$ synapses). Red, CNQX ( $n=287$ synapses). Green, CNQX plus rapamycin ( $n=746$ synapses). Blue, Rapamycin alone ( $n=566$ synapses). Evoked changes in vglut-pHluorin fluorescence intensity were significantly $\left({ }^{*} p<0.05\right.$, relative to vehicle controls at peak fluorescence levels at immediate termination of stimulus train) enhanced by treatment with CNQX, and this effect was blocked by concurrent treatment with the mTORC1 inhibitor rapamycin.

AMPAR blockade, we found that such activation was essential for the development of homeostatic changes in presynaptic function. Thus, both rapamycin and C-401 completely suppressed the increase in mEPSC frequency induced by AMPAR blockade (Fig. $2 C, E)$. Similar to AMPAR blockade, chronic $(>24 \mathrm{~h})$ treatment with L-type $\mathrm{Ca}^{2+}$ channel inhibitors induces compensatory enhancement of presynaptic and postsynaptic function (Thiagarajan et al., 2005; Gong et al., 2007), raising the question of whether mTORC1 plays a similar role during homeostatic plasticity induced by loss of L-type channel function. Indeed, we found that blocking L-type channels with $10 \mu \mathrm{M}$ nifedipine over a $3 \mathrm{~h}$ period induced a significant increase in both mEPSC amplitude (mean \pm SEM mEPSC amplitude: controls, $13.15 \pm 0.60 \mathrm{pA}, n=11$ cells; $3 \mathrm{~h}$ nifedipine, $15.38 \pm 0.70 \mathrm{pA}, n=14$ cells; $p<0.05$; data not shown) and frequency (mean \pm SEM mEPSC frequency: controls, $1.99 \pm$ $0.33 \mathrm{~Hz}$; $3 \mathrm{~h}$ nifedipine, $4.82 \pm 0.75 \mathrm{~Hz} ; p<0.05$; data not shown). As we observed with AMPAR blockade, coincident inhibition of
mTORC1 with $100 \mathrm{~nm}$ rapamycin did not affect the compensatory increase in mEPSC amplitude ( $3 \mathrm{~h}$ nifedipine plus rapamycin, $15.76 \pm 0.94 \mathrm{pA}, n=10$ cells; $p<0.05$ vs control), but strongly suppressed the increase in mEPSC frequency ( $3 \mathrm{~h}$ nifedipine plus rapamycin, $2.35 \pm 0.30 \mathrm{~Hz}$; not significant vs control) induced by nifedipine treatment. Thus, during homeostatic changes driven by either AMPAR blockade or loss of L-type $\mathrm{Ca}^{2+}$ channel function, mTORC1 inhibition selectively suppresses compensatory enhancement of mEPSC frequency, but not mEPSC amplitude.

To ensure that these mTORC1-dependent changes in mEPSC frequency following AMPAR blockade reflect compensatory enhancement of presynaptic function, we visualized actively releasing synaptic terminals by brief (5 min) live-labeling with an antibody targeted against the lumenal domain of synaptotagmin 1 (syt-lum) followed by fixation and labeling with an antibody against the vesicular glutamate transporter, vglut 1, to mark excitatory terminals. To estimate relative presynaptic function under control conditions and following AMPAR blockade, we measured the proportion of vglut1-positive terminals with detectable syt-lum staining under conditions of spontaneous vesicle release (15 min pretreatment with $2 \mu \mathrm{M}$ TTX before labeling). Using this unambiguous readout of presynaptic function, we found that neither rapamycin nor C-401 altered basal syt-lum uptake, but each prevented the increase in syt-lum uptake induced by AMPAR blockade (Fig. 3A,B). To confirm these observations and assess whether mTORC1 is necessary for homeostatic changes in action potential-driven vesicle release, we directly visualized evoked glutamate release by imaging activitydependent changes in vglut1-pHluorin fluorescence at presynaptic terminals identified by coexpression of mCherrytagged synaptophysin (Fig. 3C). As previously reported (Voglmaier et al., 2006), basal vglut1-pHluorin fluorescence at presynaptic terminals was low due to effective quenching by the acidic environment of synaptic vesicles, allowing for optical detection of action potential-triggered synaptic vesicle fusion when the lumen of the vesicle is exposed to the neutral extracellular space. To induce action potentials across the network, we applied field stimulation via parallel platinum-iridium electrodes under conditions where individual pulses each faithfully produced an action potential, verified by the characteristic cell-wide $\mathrm{Ca}^{2+}$ transient accompanying each stimulus (data not shown). In control (vehicle-treated) neurons, a $10 \mathrm{~s}$ train of action potentials delivered at $10 \mathrm{~Hz}$ induced a clear increase in vglut1-pHluorin 
A

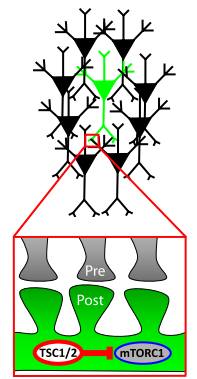

D

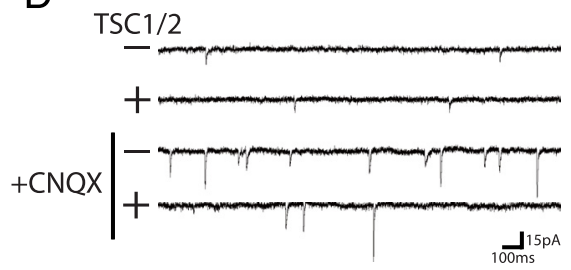

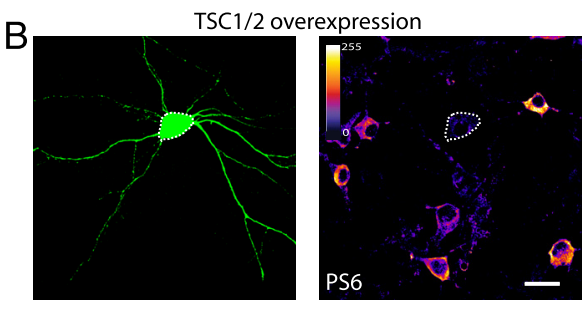

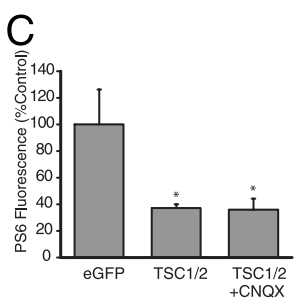

E

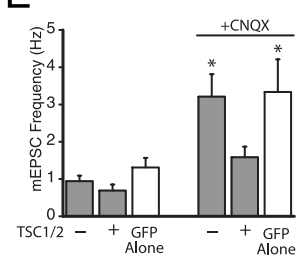

$\mathrm{F}$

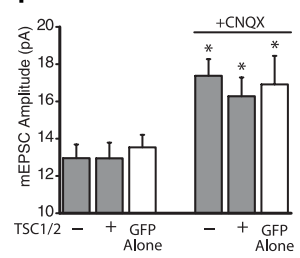

Figure 4. Postsynaptic mTORC1 activation is required for retrograde enhancement of presynaptic function. $\boldsymbol{A}$, Experimental rationale: low-efficiency transfection using calcium phosphate yields isolated transfected neurons surrounded by nontransfected cells. During recordings from TSC1/2(+) cells, mTORC1 signaling is impaired postsynaptically, while presynaptic mTORC1 activity is preserved (red insert). $\boldsymbol{B}$, Example of a TSC1/2-overexpressing neuron in a network of untransfected neurons depicting GFP (cotransfected with TSC1/2) and PS6 expression, as indicated; PS6 fluorescence intensity indicated by color look-up table. Scale bar, $40 \mu \mathrm{m}$. PS6 expression in the transfected neuron is substantially reduced relative to surrounding cells. C, Mean (+ SEM) normalized PS6 expression in transfected neurons. TSC1/2 overexpression ( $n=19$ neurons) significantly $\left({ }^{*} p<0.05, t\right.$ test) reduced basal PS6 levels compared with transfection with EGFP alone ( $n=11$ cells), and also blocked increases in PS6 by CNQX ( $n=16$ cells); ${ }^{*} p<0.05$ versus EGFP alone. $\boldsymbol{D}-\boldsymbol{F}$, Representative mEPSC recordings $(\boldsymbol{D})$ and mean $(+\boldsymbol{S E M})$ mEPSC amplitude $(\boldsymbol{E})$ and frequency $(\boldsymbol{F})$ in neurons cotransfected with TSC1/2 and EGFP $(n=11)$ with $(n=15)$ or without $(n=11)$ treatment with CNQX ( $40 \mu \mathrm{m}, 3 \mathrm{~h})$; mEPSCs in these neurons were compared with those from untransfected neighboring cells as well as from neurons from sister cultures transfected with EGFP alone. Cells were transfected at DIV $21-25$ and then used for recordings $24 \mathrm{~h}$ later. Postsynaptic inhibition of mTORC1 signaling via overexpression of TSC1/2 blocked the increase in mEPSC frequency but not the increase in mEPSC amplitude induced by AMPAR blockade. For the groups listed left to right in E: $n=8,11,16,15,11,5$ cells, respectively. ${ }^{*} p<0.05$ versus control.

fluorescence at presynaptic terminals, but following AMPAR blockade ( $40 \mu \mathrm{M}$ CNQX, $3 \mathrm{~h}$ ), this evoked vesicle fusion was markedly enhanced (Fig. 3C,D). Blocking mTORC1 activation during AMPAR blockade (100 nM rapamycin) prevented this enhancement of evoked release, suggesting that, similar to effects on spontaneous neurotransmitter release, mTORC1 activation is necessary for the enhancement of evoked release following AMPAR blockade. Together, these findings suggest that mTORC1 is specifically required for homeostatic changes in presynaptic function induced by loss of AMPAR-mediated synaptic activity.

\section{Homeostatic enhancement of presynaptic function is dependent on postsynaptic mTORC1 activation}

The fact that AMPAR blockade elicits MTORC1 activation in dendrites (Fig. $1 H, I$ ) raises the possibility that postsynaptic mTORC1 activation is necessary to drive retrograde compensation in presynaptic terminals. To address this issue, we overexpressed the tuberous sclerosis complex proteins TSC1 and TSC2, which together act as endogenous suppressors of mTORC1 activation (Inoki et al., 2002, 2003). To examine the specific role of postsynaptic mTORC1 activation, we used a low-efficiency strategy where $<1 \%$ of neurons are transfected. This enabled us to examine selective mTORC1 inhibition in single transfected neurons that receive synapses from untransfected cells with unperturbed mTORC1 signaling (Fig. $4 A)$. As expected, phosphorylation of S6, downstream of mTORC1 activity, was substantially reduced in neurons transfected with TSC $1 / 2$ relative to both untransfected neighboring neurons (Fig. $4 B$ ) and neurons transfected with EGFP alone (Fig. 4C). Moreover, TSC1/2 expression also prevented mTORC1 activation induced by AMPAR blockade (Fig. 4C). We next examined the effects of TSC $1 / 2$ expression on synap- tic compensation. Consistent with our previous observations, postsynaptic knockdown of mTORC1 activation did not alter the ability of AMPAR blockade to drive postsynaptic compensation, as similar increases in mEPSC amplitude were evident in both TSC1/2-expressing neurons and untransfected neighboring control neurons after treatment with $40 \mu \mathrm{M}$ CNQX $(3 \mathrm{~h}$, added 21 h post-transfection; Fig. $4 D, E$ ). However, the enhancement of mEPSC frequency accompanying AMPAR blockade was strongly inhibited in TSC1/2-expressing neurons relative to both untransfected controls and neurons in sister cultures transfected with EGFP alone (Fig. $4 F)$. Importantly, TSC1/2 overexpression for $24 \mathrm{~h}$ did not alter basal mEPSC frequency or amplitude in the absence of AMPAR blockade (Fig. 4D-F). Because the increase in mEPSC frequency accompanying AMPAR blockade is known to reflect an enhancement in release probability in presynaptic terminals synapsing on the recorded neuron (Jakawich et al., 2010), these results suggest that postsynaptic mTORC1 activation is necessary for retrograde changes in presynaptic function driven by AMPAR blockade.

\section{Local mTORC1 activation in dendrites is necessary for retrograde changes in presynaptic function}

We next examined whether local mTORC1 signaling in dendrites is necessary for retrograde changes in presynaptic function. To address this possibility, we used restricted microperfusion of rapamycin (200 nM) either alone or in conjunction with AMPAR blockade (bath $40 \mu \mathrm{M}$ CNQX, $120 \mathrm{~min}$; Fig. $5 A$ ), and examined subsequent live syt-lum uptake as a readout of presynaptic changes. We found that spatially restricted inhibition of mTORC1 signaling during AMPAR blockade induced a selective inhibition of syt-lum uptake at terminals apposed to the treated dendritic segment (Fig. $5 B, C)$, while vglut1 immunofluorescence in those same seg- 
A
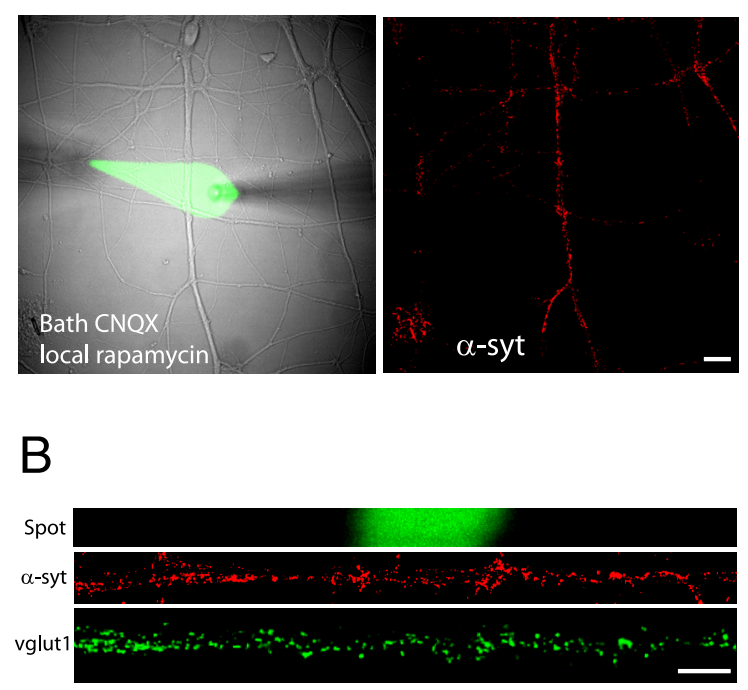

C
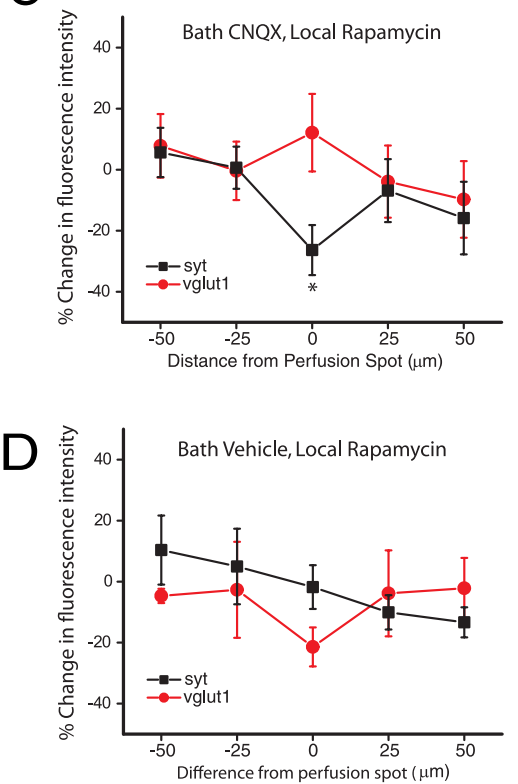

Figure 5. mTOR acts locally in dendrites to modulate presynaptic function after AMPAR blockade. $A$, Representative differential interference contrast image of a cultured hippocampal neuron exposed to AMPAR blockade ( 90 min bath application of $40 \mu \mathrm{M}$ (NQX) with superimposed rapamycin ( $200 \mathrm{~nm}$ ) perfusion spot (green) and the same neuron after staining with $\alpha$-syt. $\boldsymbol{B}$, Linearized primary dendrite from the cell shown in $\boldsymbol{A}$ with corresponding $\alpha$-syt and vglut1 staining registered to the perfusion area (green). Scale bars: $25 \mu \mathrm{m}$. A decrease in syt-lum uptake is evident in the perfused area, whereas vglut1 expression remains stable. $\boldsymbol{C}, \boldsymbol{D}$, Mean ( \pm SEM) normalized syt-lum/vglut1 staining in treated and untreated dendritic segments from cells subject to local microperfusion of rapamycin ( $200 \mathrm{~nm}$ ) with or without concurrent AMPAR blockade; all data are expressed as a percentage change in fluorescence relative to the average value in untreated segments. Local rapamycin perfusion significantly $\left({ }^{*} p<0.05\right)$ decreased syt-lum uptake in the treated area relative to vglut1 expression in the same dendritic segment when $\mathbf{C N Q X}$ was present $(\boldsymbol{C})$ ( $n=10$ dendrites from 10 neurons), but not when vehicle was applied to the bath $(\boldsymbol{D})$ ( $n=4$ dendrites from 4 neurons).

ments was not significantly altered. By contrast, local rapamycin treatment in the absence of AMPAR blockade (Fig. 5D) did not alter syt-lum uptake, demonstrating a specific role for local mTORC1 signaling in presynaptic compensation induced by AMPAR blockade. Importantly, the brief ( $5 \mathrm{~min}$ ) syt-lum labeling period used in these microperfusion experiments argues against a major influence from the transit of synaptic vesicles between different presynaptic terminals. Indeed, we observed only minimal fluorescence recovery over a $50 \mathrm{~min}$ period after locally photobleaching syt-lum signal (data not shown), indicating that the signal observed in treated dendritic segments under brief labeling conditions primarily reflects uptake at synapses apposed to those segments. These results thus suggest that mTORC1 activation in dendrites plays a spatially restricted role in driving retrograde homeostatic changes in presynaptic function in response to AMPAR blockade.

\section{mTORC1 operates upstream of BDNF synthesis during} homeostatic plasticity

Postsynaptic release of BDNF is necessary for retrograde changes in presynaptic function driven by AMPAR blockade (Jakawich et al., 2010; Lindskog et al., 2010), raising the question of whether mTORC1 operates upstream or downstream of BDNF action. Indeed, exogenous BDNF application is known to activate mTORC1 signaling (Schratt et al., 2004), raising the possibility that mTORC1 activation in the context of synaptic attenuation might be secondary to BDNF release. However, contrary to this notion, we found that mTORC1 activation induced by AMPAR blockade was BDNFindependent as scavenging extracellular BDNF with TrkB-Fc $(1 \mu \mathrm{g} /$ $\mathrm{ml}$ ) did not affect phosphorylation of $\mathrm{mTORC} 1$ effectors in response to CNQX treatment (Fig. 6A,B). To explore this issue further, we examined whether presynaptic changes driven by direct BDNF application were sensitive to coincident mTORC1 inhibition with rapamycin. As shown in Figure 6C,D, application of BDNF (250 $\mathrm{ng} / \mathrm{ml}$ ) to cultured neurons for $60 \mathrm{~min}$ induced a sustained increase in mEPSC frequency that remained persistent following BDNF washout (mEPSCs were recorded 30-50 min following BDNF removal). Importantly, direct BDNF application induced a similar increase in $\mathrm{mEPSC}$ frequency in the presence or absence of rapamycin (Fig. $6 C, D$ ), indicating that BDNF modulates presynaptic function in an mTORC1-independent fashion. Likewise, BDNF-induced enhancement of syt-lum uptake at vglut1-positive excitatory terminals was similarly unaffected by coincident rapamycin treatment (Fig. $6 E, F)$, indicating that BDNF acts downstream of mTORC1 activation to drive enhancement of presynaptic function. Given the role of mTORC1 in translational control, these findings suggest that mTORC1 activation might participate in the regulation of de novo BDNF synthesis, which is necessary for retrograde homeostatic changes in presynaptic function (Jakawich et al., 2010). Consistent with this possibility, Western blots of hippocampal neuron lysates revealed that the increase in BDNF expression in response to AMPAR blockade was prevented by rapamycin (Fig. 7A,B). To assess the cellular compartments where this mTORC1-dependent regulation of BDNF occurs, we examined BDNF expression by immunocytochemistry. Consistent with earlier reports (Jakawich et al., 2010), AMPAR blockade induced a significant increase in BDNF expression in dendrites without altering somatic BDNF levels (Fig. $7 C, D)$. This highly compartmentalized increase in dendritic BDNF expression induced by AMPAR blockade was prevented by $\mathrm{mTORC1}$ inhibition with rapamycin. These findings suggest that $\mathrm{mTORC1}$ is a critical regulator of new BDNF synthesis following AMPAR blockade. 
Cell-autonomous enhancement of synaptic activity accompanies postsynaptic mTORC1 activation We next asked whether postsynaptic mTORC1 activation in the absence of AMPAR blockade is sufficient for driving retrograde changes in presynaptic function. To examine this question, we again used a sparse expression strategy while transfecting the mTORC1 activating GTPase Rheb (data not shown) or a hyperactive RhebQ64L mutant (Fig. 8A) that is persistently in its GTP-bound state (Jiang and Vogt, 2008). In non-neuronal cells, expression of Rheb or RhebQ64L is sufficient to drive sustained cell-autonomous mTORC1 signaling (Tee et al., 2003; Long et al., 2005). Likewise, $24 \mathrm{~h}$ following transfection, immunocytochemistry against PS6 confirmed robust dendritic and somatic mTORC1 activation in RhebQ64Ltransfected neurons relative to either surrounding untransfected neurons (Fig. $8 B$ ) or neurons from sister cultures transfected with EGFP alone (Fig. 8C). To examine the impact of cell-autonomous mTORC1 activation on overall excitatory synaptic drive in active networks, we compared spontaneous excitatory synaptic activity in RhebQ64L-expressing neurons with neighboring untransfected neurons in the same culture and EGFP-transfected neurons in sister cultures in the absence of TTX. Recording spontaneous EPSCs in the presence of bicuculine and APV, we found that overall excitatory network activity was significantly enhanced onto RhebQ64Lexpressing neurons, reflected in $>5$-fold increase in charge transfer relative to untransfected neurons in the same culture (Fig. 8D,E). To examine whether these changes in synaptic efficacy derive from growth of new synaptic connections, we examined the density of dendritic spines in response to either chronic suppression (with TSC1/2 overexpression) or hyperactivation (with RhebQ64L expression) of mTORC1. We found that alteration of mTORC1 signaling for $\sim 24 \mathrm{~h}$ or $5 \mathrm{~d}$ failed to alter the density of dendritic spines (Fig. $8 \mathrm{~F}-\mathrm{H}$ ) relative to control neurons expressing GFP alone, despite clear mTORCdependent regulation of cell soma size ( $5 \mathrm{~d})$. While it is likely that more prolonged changes in mTORC1 signaling are sufficient to alter synaptic growth, these results suggest that the cell-autonomous increase in synaptic efficacy observed here results largely from altered function of existing synaptic connections.

\section{Postsynaptic mTORC1 activation is sufficient to drive} retrograde changes in presynaptic function

We next examined the mechanisms by which postsynaptic mTORC1 activation leads to enhanced synaptic efficacy. Given that the enhancement of dendritic BDNF expression following AMPAR blockade is dependent on mTORC1 (Fig. 7C,D), we first assessed whether

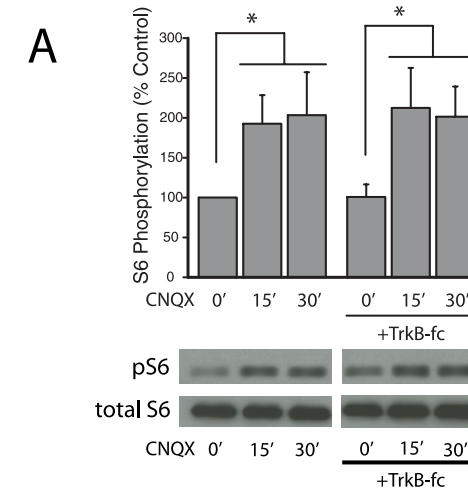

B
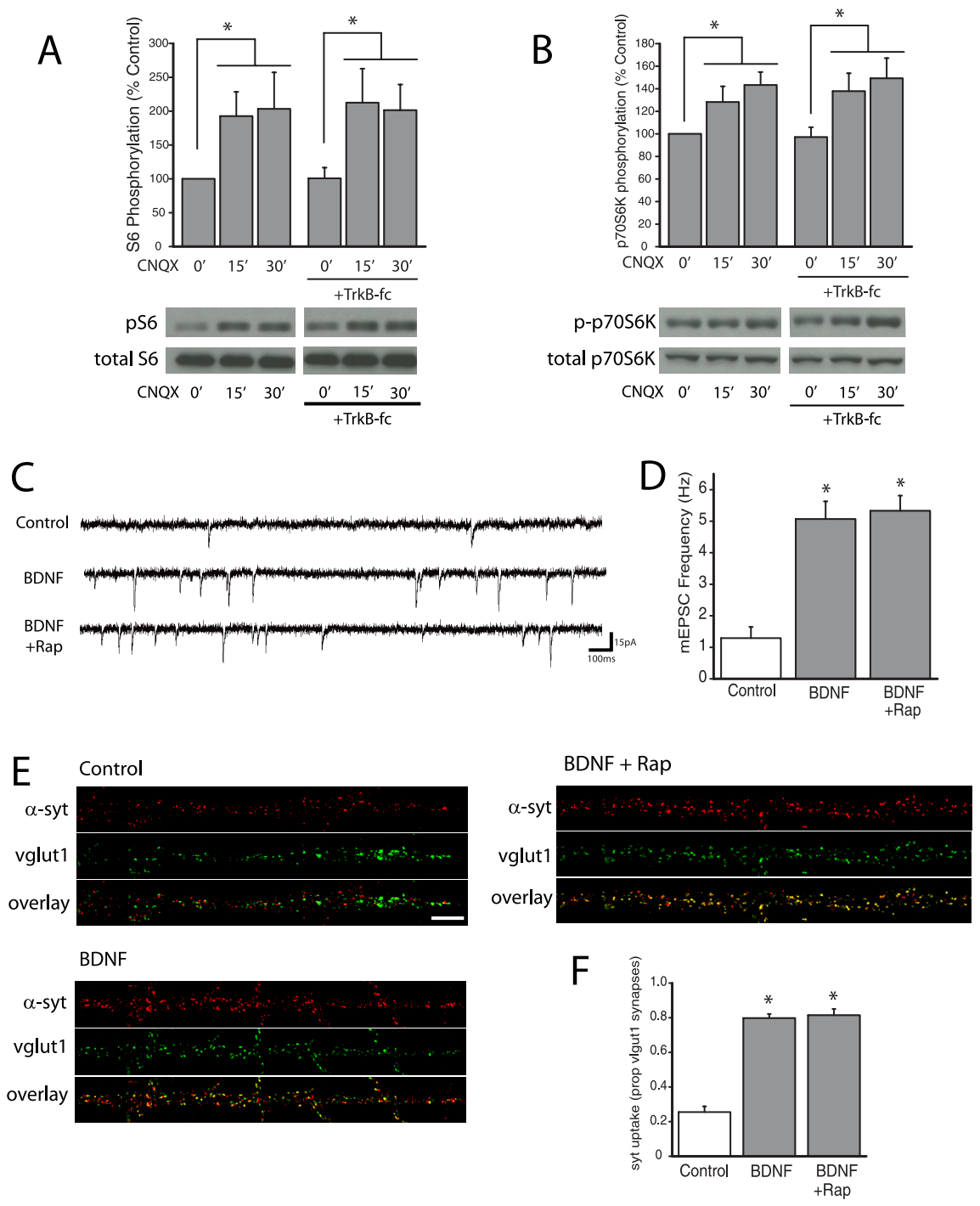

Figure 6. BDNF enhances presynaptic function downstream of mTORC1. $A, B$, Representative Western blots and summary data from experiments $(n=5)$ examining the phosphorylation of downstream mTORC1 effectors $\$ 6(\boldsymbol{A})$ and p70S6K $(\boldsymbol{B})$ during AMPAR blockade. Cultured neurons were treated with CNQX (40 $\mu \mathrm{M})$ with or without TrkB-Fc $(1 \mu \mathrm{g} / \mathrm{ml}, 30 \mathrm{~min}$ pretreatment) before ot affected by scavenging extracellular BDNF. $\boldsymbol{C}, \boldsymbol{D}$, Representative recordings $(\boldsymbol{C})$ and mean $(+$ SEM) $\mathrm{mEPSC}$ frequency $(\boldsymbol{D})$ after tative examples $(\boldsymbol{E})$ and mean ( + SEM) syt-lum uptake $(\boldsymbol{F})$ from experiments $(n=15 /$ group) where neurons were treated with BDNF ( $250 \mathrm{ng}, 60 \mathrm{~min}$ ) with or without rapamycin ( $100 \mathrm{~nm} ; 30 \mathrm{~min}$ before BDNF). Inhibiting mTORC1 signaling did not impact enhanced syt-lum uptake induced by BDNF treatment $\left({ }^{*} p<0.05\right.$, relative to control). Scale bar: $\boldsymbol{E}, 10 \mu \mathrm{m}$.

postsynaptic $\mathrm{mTORC1}$ activation is sufficient to alter BDNF expression in the cell body or dendritic compartment of hippocampal neurons. Indeed, RhebQ64L-expressing neurons exhibited a striking increase in dendritic BDNF expression without altering MAP2 levels in the same neurons (Fig. 9A,B). Moreover, the pattern of changes following $\mathrm{mTORC1}$ activation was highly reminiscent of changes in BDNF expression that accompany AMPAR blockade (Fig. 7C,D), as the change in BDNF expression was again highly compartmentalized, with no significant alteration in somatic BDNF levels (Fig. $9 A, B)$. A strikingly similar pattern was observed with overexpression of wild-type Rheb, where dendritic BDNF expression increased significantly (mean \pm SEM percentage change in BDNF fluorescence relative to GFP expression alone, $+145.7 \pm 26.1$; 
A
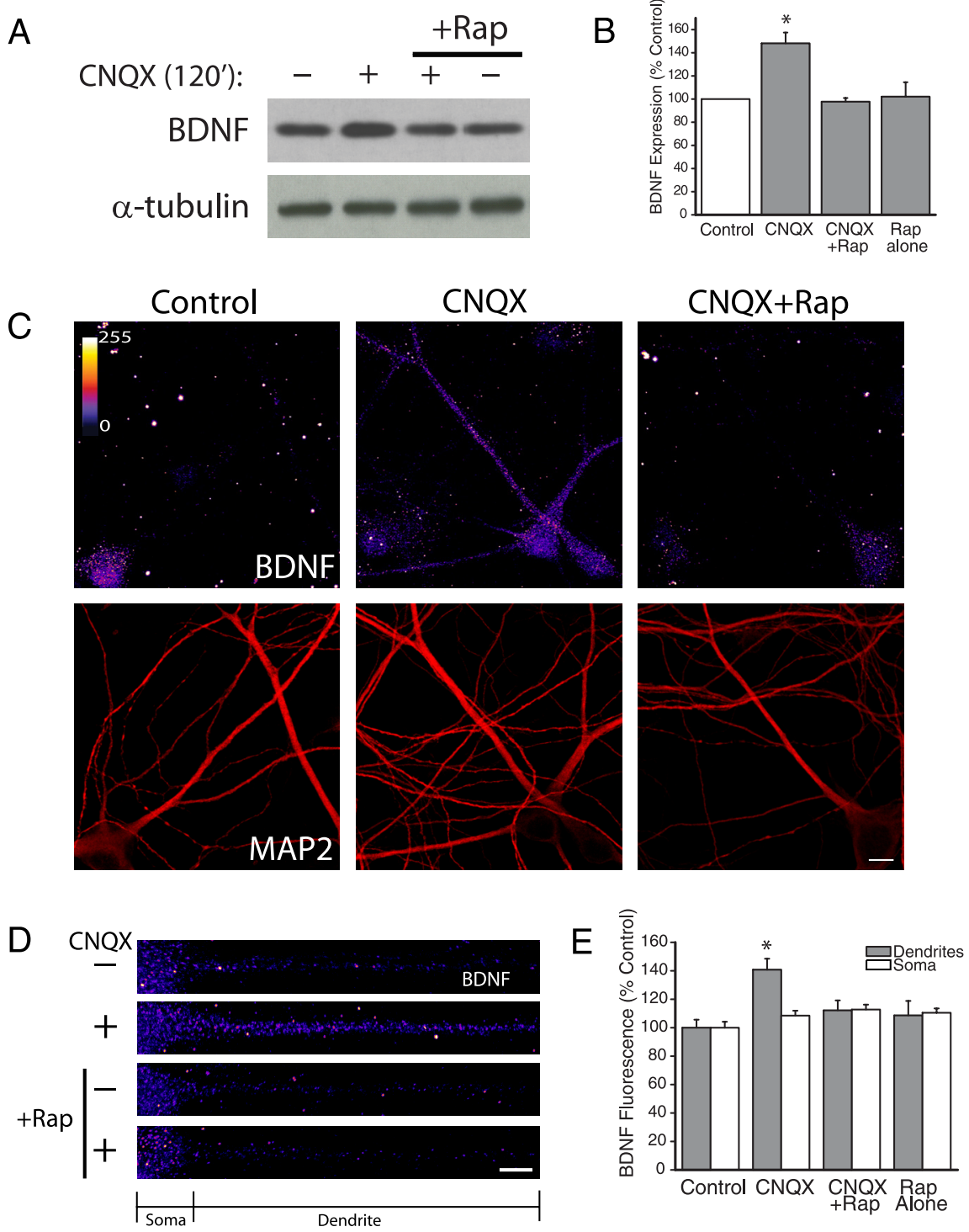

Figure 7. Compartmentalized enhancement in BDNF expression by AMPAR blockade requires mTORC1. $A, B$, Representative Western blots $(\boldsymbol{A})$ and summary data $(\boldsymbol{B})$ in experiments $(n=6)$ where neurons were treated with CNQX ( $40 \mu \mathrm{m}, 120 \mathrm{~min})$ with or without rapamycin (100 nm, 30 min pretreatment) before harvesting; ${ }^{*} p<0.05$ relative to control. The BDNF band ( $\left.\sim 14 \mathrm{kDa}\right)$ represents mature BDNF. C, $\boldsymbol{D}$, BDNF expression in linearized somatic and dendritic segments and mean ( + SEM) expression BDNF in somatic and dendritic compartments normalized to average control values, in neurons treated with CNQX ( $40 \mu \mathrm{M}, 3 \mathrm{~h}$ ) with or without rapamycin ( $100 \mathrm{~nm}, 30$ min pretreatment). E, AMPAR blockade significantly enhanced BDNF expression in dendrites ( ${ }^{*} p<$ $0.05, n=30$ neurons) compared with untreated control neurons $(n=33)$. This effect was blocked by pretreatment with rapamycin (100 nm; $n=31$ neurons), which had no effect on BDNF expression on its own ( $n=29$ neurons). Somatic BDNF expression from these same cells did not change across treatment groups. Scale bar: $C, 10 \mu \mathrm{m}$.

$p<0.05$; data not shown) without concomitant changes in steady-state BDNF expression in the cell body (mean \pm SEM percentage change in BDNF fluorescence relative to GFP expression alone, $-13.4 \pm 5.1$, not significant; data not shown). These selective changes in dendritic BDNF expression are not due to compartment-selective mTORC1 activation, as expression of both Rheb (data not shown) and RhebQ64L (Fig. 8 B) enhance PS6 staining in both the cell bodies and dendrites. These observations suggest that dendritic and somatic expression of BDNF is subject to distinct compartment-specific regulatory control.

To determine how postsynaptic mTORC1 activation alters synaptic efficacy and to what extent the changes in BDNF expression contribute, we recorded mEPSCs from RhebQ64L-transfected neurons or neighboring untransfected control neurons with unaltered
mTORC1 signaling. The sparse transfection conditions gave rise to isolated RhebQ64Lexpressing neurons that receive nearly all their synaptic inputs from untransfected neurons with unaltered $\mathrm{mTORC1}$ signaling (Fig. $8 \mathrm{~A}$ ), enabling us to evaluate the effects of postsynaptic mTORC1 activation on both presynaptic and postsynaptic function. Despite the strong increase in synaptic drive induced by postsynaptic RhebQ64L expression ( $24 \mathrm{~h}$ post-transfection), the amplitude of mEPSCs onto these neurons was not altered relative to neighboring untransfected neurons (Fig. $9 C, D$ ) or neurons expressing GFP alone (Fig. 4E). However, RhebQ64Lexpressing neurons exhibited a marked increase in mEPSC frequency (Fig. 9C-E), suggesting enhanced spontaneous release from synaptic terminals that impinge on dendrites with activated MTORC1 signaling. Like the changes induced by AMPAR blockade, these retrograde changes in presynaptic function driven by postsynaptic mTORC1 activation required BDNF release, as they were completely prevented by the extracellular BDNF scavenger TrkB-Fc $(1 \mu \mathrm{g} / \mathrm{ml}$; Fig. $9 C-E)$. A very similar pattern of results was observed with postsynaptic expression of wild-type Rheb in parallel experiments, where postsynaptic Rheb expression induced a significant increase in $\mathrm{mEPSC}$ frequency (mean $\pm \mathrm{SEM}$ mEPSC frequency, nontransfected controls: $1.52 \pm 0.17 \mathrm{~Hz}$; Rheb transfected neurons: $2.35 \pm 0.40 \mathrm{~Hz}, p<0.05$ onetailed; data not shown) without altering mEPSC amplitude (mean \pm SEM mEPSC amplitude, nontransfected controls: $13.95 \pm 0.66 \mathrm{pA}$; Rheb transfected neurons: $14.03 \pm 0.65 \mathrm{pA}$, not significant; data not shown). As with RhebQ64L, the increase in mEPSC frequency with Rheb overexpression is similarly abolished by scavenging extracellular BDNF (mean \pm SEM mEPSC frequency, nontransfected controls with TrkB-Fc: $1.51 \pm 0.19 \mathrm{~Hz}$; Rheb transfected neurons: $1.29 \pm 0.15 \mathrm{~Hz}$; data not shown).

To examine whether a similar enhancement of evoked neurotransmission is also established by postsynaptic mTORC1, we examined a use-dependent block of evoked NMDAR-mediated EPSCs onto RhebQ64L-expressing neurons and untransfected neurons from the same cultures with the open-channel blocker MK-801 to assess neurotransmitter release probability (Hessler et al., 1993; Rosenmund et al., 1993). Immediately before recording, evoked NMDAR EPSCs were pharmacologically isolated and elicited at a rate of $0.33 \mathrm{~Hz}$ with an extracellular stimulating electrode positioned near the recording neuron. Before MK-801 treatment, the kinetics of evoked NMDAR EPSCs in RhebQ64Lexpressing neurons, untransfected neurons, and control GFPexpressing neurons were similar (mean \pm SEM time to $50 \%$ decay, nontransfected controls: $75.40 \pm 9.34 \mathrm{~ms}, n=10$ cells; GFP- 
A

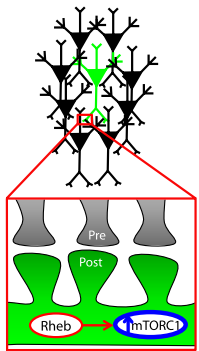

D

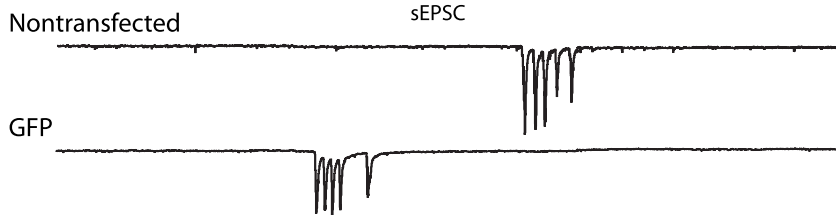

RhebQ64L

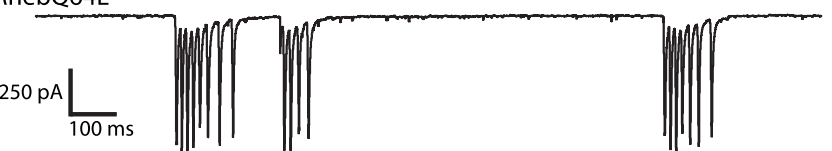

B

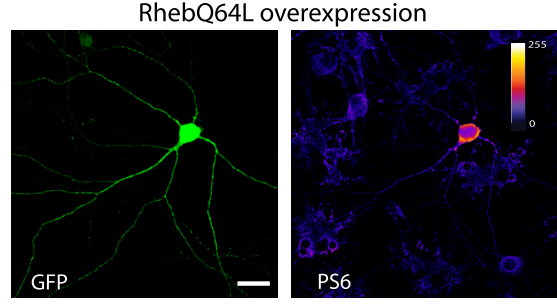

C

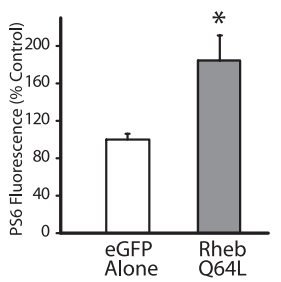

$E$

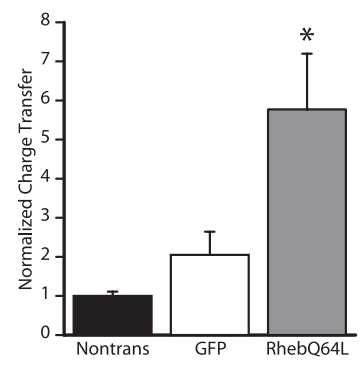

F

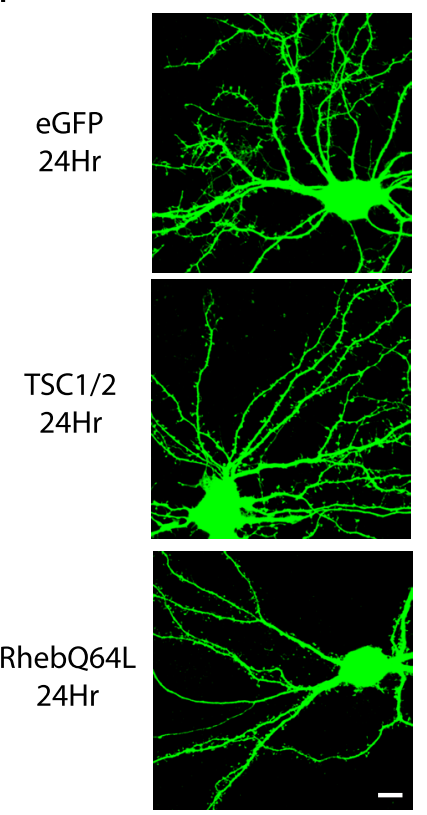

G

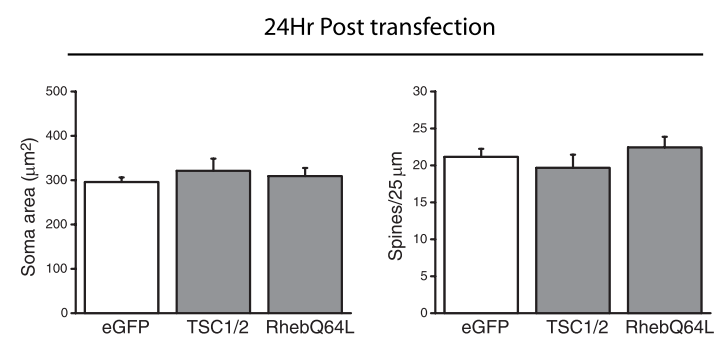

$\mathrm{H} 5$ Days Post transfection
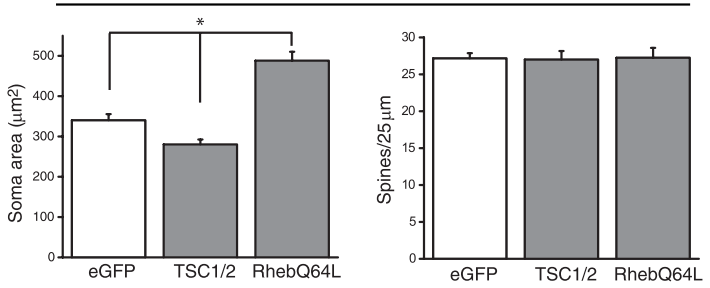

Figure 8. Sparse mTORC1 activation enhances synaptic efficacy in a cell-autonomous fashion. $A$, Experimental rationale: Low-efficiency transfection using calcium phosphate yields individual transfected neurons surrounded by nontransfected cells. During recordings from RhebQ64L (+) cells, mTORC1 signaling is enhanced postsynaptically, while presynapticmTORC1 activity is normal (red insert). B, C, Example images $(\boldsymbol{B})$ and mean ( + SEM) normalized PS6 expression ( $\left(\right.$ ) in transfected neurons. Expression of RhebQ64L ( $n=14$ cells) significantly ( ${ }^{*} p<0.05$ vs EGFP alone by t test) enhanced PS6 levels compared with transfection with EGFP alone ( $n=14$ cells). PS6 fluorescence intensity indicated by color look-up table. Scale bar, $40 \mu \mathrm{m}$. $\boldsymbol{D}, \boldsymbol{E}$, Representative recordings of sEPSCs $(\boldsymbol{D})$ and mean charge transfer $(\boldsymbol{E})$ of RhebQ64L-expressing neurons $(n=17)$ or neighboring nontransfected neurons $(n=16)$, as well as EGFP-expressing neurons $(n=19)$ in low $\mathrm{Ca}^{2+}(0.5 \mathrm{~mm})$ extracellular solution. Quantificative analysis of spontaneous activity was performed by measuring the total charge transfer in 20 s relative to nontransfected cells. Postsynaptic expression of RhebQ64L was sufficient to induce an increase in spontaneous activity of nontransfected presynaptic neurons. ${ }^{*} p<0.01$, one-way ANOVA, Tukey-Kramer post hoc. $\boldsymbol{F}-\boldsymbol{H}$, Effects of altered mTORC1 signaling on cell growth pathways in hippocampal neurons. $\boldsymbol{F}$, Representative images of cultured hippocampal neurons cotransfected with GFP as well as either TSC1/2 or RhebQ64L. Scale bar, $10 \mu \mathrm{m}$. G, H, After $24 \mathrm{~h}$, neither TSC1/2 $(n=21)$ nor RhebQ64L overexpression ( $n=16)$ has any effect on soma size or spine density when compared with cells expressing EGFP alone $(n=71)$. In contrast, $5 \mathrm{~d}$ of TSC1/2 expression yields significantly diminished soma size ( $n=47)$ while prolonged RhebQ64L expression ( $n=27$ ) results in significantly enhanced soma size compared with control EGFP-expressing cells $(n=30)$; again, no significant change in spine density was observed.

expressing neurons: $81.72 \pm 9.15 \mathrm{~ms}, n=11$ cells; RhebQ64Lexpressing neurons: $85.09 \pm 7.75 \mathrm{~ms}, n=9$ cells; $F_{(2,27)}=0.29$, not significant), suggesting similar NMDAR subunit composition in the three conditions. As expected for an increase in release probability, we found that MK-801 blockade of NMDAR currents was signifi- cantly accelerated in RhebQ64L-expressing neurons relative to untransfected neurons from the same cultures, as well as from control neurons expressing GFP in sister cultures (Fig. 9F,G). Again, the extracellular BDNF scavenger TrkB-Fc completely eliminated the enhanced release probability onto RhebQ64L-expressing neurons, 

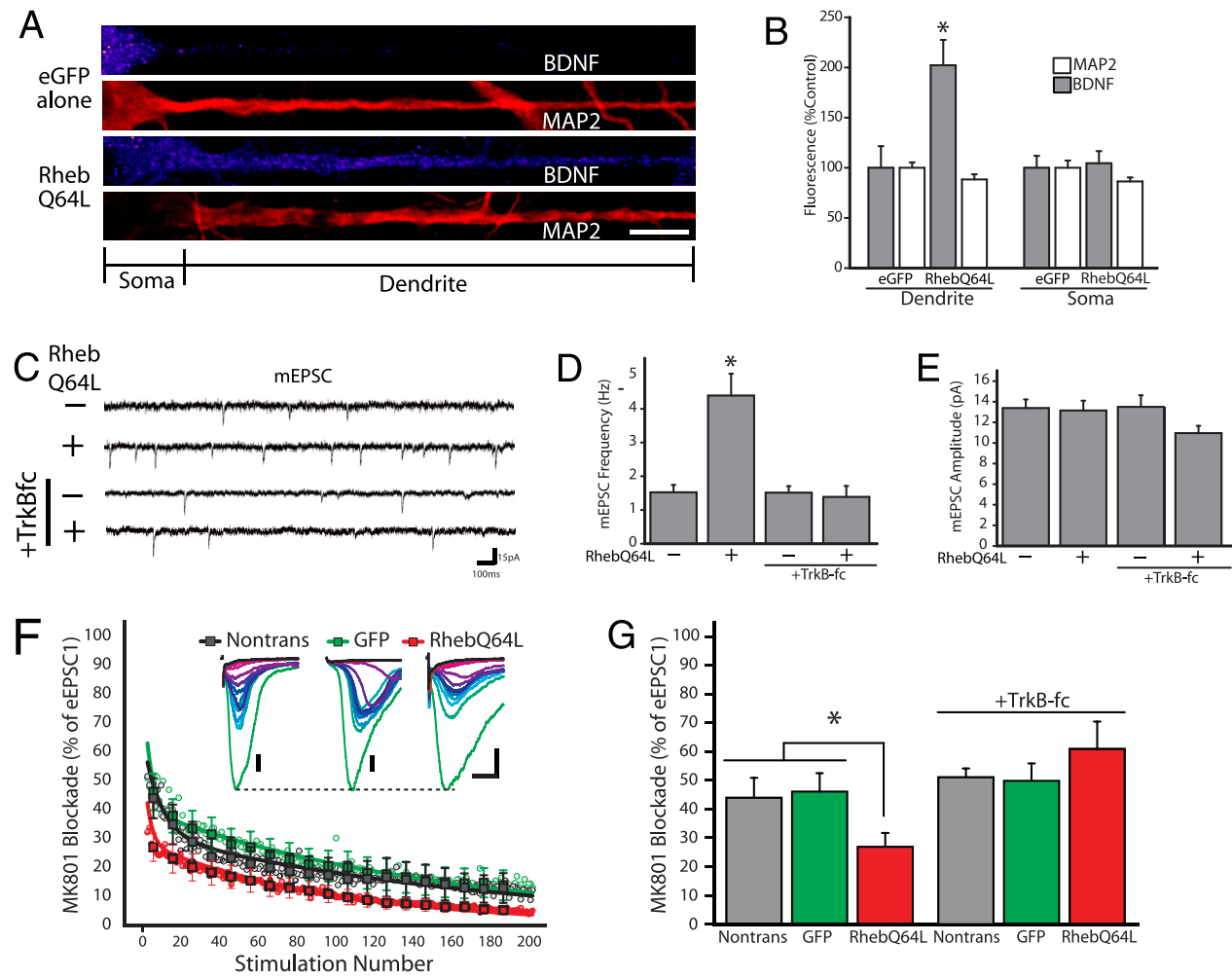

Figure 9. Postsynaptic mTORC1 activation drives enhancement of presynaptic function via BDNF release. $\boldsymbol{A}, \boldsymbol{B}, \mathrm{BDNF}$ and MAP2 expression in linearized somatic and dendritic segments $(\boldsymbol{A})$, and mean (+SEM) expression (B) of MAP2 and BDNF in somatic and dendritic compartments normalized to average control values, in neurons cotransfected with RhebQ64L and EGFP or neurons transfected with EGFP alone. While BDNF expression in dendrites was significantly ( ${ }^{*} p<0.05$, relative to EGFP alone) enhanced by expression of RhebQ64L ( $\left.n=22\right)$ compared with control neurons expressing EGFP alone $(n=22)$, somatic expression from these same cells was unchanged. MAP2 expression did not differ between groups. Scale bar: $\boldsymbol{A}, 10 \mu \mathrm{m}$. $(-\boldsymbol{E}$, Representative recordings $(\boldsymbol{C})$ and mean (+SEM) mEPSC frequency $(\boldsymbol{D})$ and amplitude $(\boldsymbol{E})$ in nontransfected neurons $(n=9)$ or neurons transfected with RhebQ64L as well as EGFP $(n=7)$ with or without treatment with TrkB-fc $(1 \mu \mathrm{g} / \mathrm{ml}$ applied immediately post-transfection: RhebQ64L( -$)$ with TrkB-fc, $n=6$; RhebQ64L( + ) with TrkB-fc, $n=10)$. Cells were transfected at DIV $21-25$ and then used for recordings $24 \mathrm{~h}$ later. Postsynaptic enhancement of mTORC1 signaling via expression of RhebQ64L was sufficient to increase mEPSC frequency compared with cells expressing EGFP alone, and this effect was blocked by scavenging extracellular BDNF with TrkB-fc. Neither RhebQ64L expression nor treatment with TrkB-fc had any effect on mEPSC amplitude. ${ }^{*} p<0.05$, relative to EGFP alone. $F$, Average MK801 blockade of RhebQ64L ( $n=11), \operatorname{EGFP~}(n=11)$, and nontransfected $(n=10)$ neurons. MK801 $(20 \mu \mathrm{m})$ was applied to the bath $\left(0 \mathrm{Mg}^{2+}\right.$, with CNQX and bicuculline) for 5 min, followed by 200 extracellular stimulations $(0.33 \mathrm{~Hz})$, while voltage-clamping the cell at $-70 \mathrm{mV}$ to record NMDAR-mediated currents. The amplitude of each response was measured and expressed relative to the amplitude of the first response. The mean for each response is plotted in small circles and fitted by a double-exponential curve (untransfected: $\tau_{1}=8.64, \tau 2=175.8$; EGFP: $\tau_{1}=3.92, \tau 2=157.33$; RhebQ64L: $\left.\tau_{1}=2.93, \tau 2=96.97\right)$. G, Average percentage response of the second through eleventh stimulation relative to the first EPSC. Expression of RhebQ64L in the postsynaptic neuron was sufficient to increase the rate of MK801 blockade and extracellular incubation of TrkB-fc with RhebQ64L expression blocked the increase. ${ }^{*} p<0.01$, one-way ANOVA, Tukey-Kramer post hoc.

suggesting that postsynaptic mTORC1 activation controls evoked neurotransmitter release through the release of BDNF as a retrograde messenger. Together, these results suggest that postsynaptic mTORC1 activation is sufficient to drive retrograde enhancement of presynaptic function in a BDNF-dependent fashion, a manner similar to homeostatic changes in presynaptic function induced by loss of excitatory synaptic input.

\section{Discussion}

Our results identify a novel mode of synaptic regulation conferred by local mTORC1-dependent signaling in dendrites. While the role of $\mathrm{mTORC1}$ in contributing to alterations in postsynaptic function during protein synthesis-dependent forms of synaptic plasticity is well established (Tang et al., 2002; Cammalleri et al., 2003; Hou and Klann, 2004), here we have shown that dendritic $\mathrm{mTORC} 1$ activation also gates a local retrograde signaling mechanism that drives changes in presynaptic function from apposed postsynaptic terminals. This unique trans-synaptic role for dendritic mTORC1 signaling is critically dependent on BDNF release, which serves to couple loss of excitatory synaptic drive with retrograde compensation of presynaptic function. Finally, we show that dendritic mTORC1 plays a spatially restricted role in retrograde modulation of presynaptic function induced by
AMPAR blockade, revealing a unique role for mTORC1 as part of a homeostatic translational control pathway that operates locally in dendrites and underlies trans-synaptic control of presynaptic function.

\section{Parallel translational regulatory pathways in dendrites} mediate distinct homeostatic adaptations at synapses While alterations in postsynaptic firing have been shown to exert cell-wide homeostatic changes in synaptic strength (Ibata et al., 2008; Goold and Nicoll, 2010), several groups have demonstrated that compensatory synaptic adaptations can also be implemented locally in dendrites (Sutton et al., 2006; Aoto et al., 2008; Jakawich et al., 2010), even at individual synapses (Lee et al., 2010; Béique et al., 2011; Hou et al., 2011). Indeed, acute loss of excitatory synaptic input induces a rapid and local recruitment of GluA1 homomeric receptors to affected synapses. This recruitment is critically dependent on dendritic protein synthesis (Sutton et al., 2006; Aoto et al., 2008; Maghsoodi et al., 2008). In hippocampal neurons, this rapid postsynaptic compensation can be induced by blockade of either AMPARs or NMDARs, whereas retrograde compensation of presynaptic terminals accompanies AMPAR blockade but not NMDAR blockade (Jakawich et al., 2010). We also find that AMPAR blockade rapidly activates mTORC1 sig- 
naling in dendrites of hippocampal neurons, but NMDAR blockade over the same period does not. However, it is clear that patterned synaptic activity drives mTORC1 signaling in hippocampal neurons through an NMDAR-dependent mechanism (Tang et al., 2002; Cammalleri et al., 2003), and that loss of NMDAR activity in cortical neurons can also activate mTORC1 signaling (Li et al., 2010; Wang et al., 2011). Whether these differences reflect unique coupling of AMPARs to inactivationinduced mTORC1 signaling in hippocampal neurons or differences in the timing or context of NMDAR blockade await future studies. On the other hand, the coupling between AMPARs and mTORC1 activation that we describe in hippocampal neurons has also been recently observed at the Drosophila neuromuscular junction (NMJ). Similar to our findings, Penney and colleagues (2012) document a requirement for postsynaptic TOR activation in vivo during retrograde homeostatic plasticity driven by AMPAR blockade at the NMJ, and also find that genetic upregulation of postsynaptic TOR activity is sufficient to drive retrograde enhancement of presynaptic function. Together with our results, these findings suggest that the retrograde signaling role for mTORC1 at synapses is evolutionarily conserved.

We find that postsynaptic compensation induced by AMPAR blockade, which, in hippocampal neurons, is highly similar to that following acute loss of NMDAR activity, is insensitive to mTORC1 inhibitors or postsynaptic knockdown of mTORC1 signaling (but see Wang et al., 2011). Consistent with our results, Maghsoodi et al. (2008) found that mTORC1 inhibition with rapamycin did not disrupt postsynaptic compensation induced by retinoic acid, which is an essential intermediate in the recruitment of GluA1 homomeric receptors to synapses during NMDAR blockade (Aoto et al., 2008; Soden and Chen, 2010). These results illustrate that homeostatic plasticity of postsynaptic function differs from L-LTP and mGluR-LTD in hippocampal neurons in relying on a distinct mTORC1-independent translational control pathway in dendrites for its induction. We speculate that the dependence on unique translation regulatory events likely reflects a requirement for a distinct set of newly synthesized effector proteins in each case, despite the common regulation of synaptic AMPAR content as a mode of expression. Interestingly, we found that postsynaptic mTORC1 activation over a timeframe that encompasses L-LTP and mGluR-LTD induction was insufficient to drive changes in postsynaptic function directly. This suggests that the unique complement of postsynaptic proteins synthesized in an mTORC1-dependent fashion (Schratt et al., 2004; Slipczuk et al., 2009; Neasta et al., 2010) must interact with other activity-dependent signaling events to alter postsynaptic function in an enduring fashion. While relatively acute mTORC1 activation $(<24 \mathrm{~h})$ in our experiments did not alter postsynaptic function directly, there is evidence to suggest that longer, chronic hyperactivation of neuronal mTORC1, via knockdown of TSC1 or TSC2, is associated with morphological and functional changes in the postsynaptic compartment (Tavazoie et al., 2005; Bateup et al., 2011). Our findings raise the intriguing possibility that the alterations in postsynaptic structure and function that accompany more chronic postsynaptic mTORC1 activation might be secondary to altered neurotransmitter release from apposed synaptic terminals, given that these functional changes in the presynaptic compartment are the first to emerge following mTORC1 activation.

While the functional role of dendritic protein synthesis on postsynaptic function has been heavily studied, it is now clear that alteration of presynaptic function by dendrite-derived factors can exert an important additional level of regulatory control at synapses (Regehr et al., 2009). Such retrograde signaling is also known to play a pivotal role in synaptic homeostasis (Frank et al., 2006; Branco et al., 2008; Jakawich et al., 2010; Lindskog et al., 2010). For example, local activity in a specific dendritic branch has been shown to homeostatically modulate release probability from apposed presynaptic terminals (Branco et al., 2008), and similar retrograde changes in presynaptic function are dependent on local dendritic protein synthesis (Jakawich et al., 2010). Our results demonstrate an essential role for mTORC1 activation in this local retrograde modulation of presynaptic function. Hence, rapid homeostatic adjustment of presynaptic and postsynaptic function is subserved by parallel mTORC1-dependent and mTORC1-independent translational control pathways in dendrites.

\section{mTORC1-dependent retrograde signaling requires BDNF as a downstream effector}

We identified BDNF as a critical effector acting downstream of mTORC1 activation in driving enhancement of presynaptic function. mTORC1 signaling is necessary for enhanced BDNF expression accompanying AMPAR blockade, and postsynaptic mTORC1 activation in the absence of AMPAR blockade is sufficient to enhance dendritic BDNF expression. Given that mTORC1 is required locally in dendrites for presynaptic compensation and that AMPAR blockade drives local dendritic BDNF synthesis (Jakawich et al., 2010), our results suggest that dendritic mTORC1 likely directly controls BDNF synthesis in dendrites. Consistent with this notion, we find that mTORC1 activation induces highly compartmentalized changes in BDNF expression, with significant elevation in dendrites despite no measurable changes in the cell body from the same neurons. Presently, it is unclear what mechanisms account for the compartment-specific changes in BDNF expression accompanying mTORC1 activation, although it is known that the BDNF mRNA pool comprises up to 16-22 transcripts with unique $5^{\prime}$ and/or 3' UTRs (Aid et al., 2007). Moreover, these distinct BDNF transcripts exhibit differences in activity-dependent dendritic trafficking (Pattabiraman et al., 2005; Chiaruttini et al., 2008) as well as constitutive dendritic localization (An et al., 2008; Baj et al., 2011), raising the possibility that only a small subset of these, which may comprise a greater relative proportion of the dendritic BDNF mRNA pool (Baj et al., 2011), are responsive to mTORC1 activation (Liao et al., 2012). Future studies are needed to map the mTORC1-responsive sites (or lack thereof) in each BDNF transcript to test this idea. Another unresolved question relates to the relative influence of proBDNF and mature BDNF in driving the presynaptic changes induced by dendritic mTORC1 activation. Whereas mature BDNF is clearly upregulated by AMPAR blockade in a rapamycin-sensitive fashion (Fig. $7 A, B$ ), further studies are needed to test whether the presynaptic changes induced by dendritic mTORC1 activation require the release of proBDNF, mature BDNF, or some combination of the two.

\section{A novel target for cognitive dysfunction caused by dysregulated mTORC1 signaling?}

Several disorders resulting in cognitive impairment and mental retardation have been suggested to share dysregulation of local protein synthesis in dendrites as a common feature (Hoeffer and Klann, 2010), specifically via overactive signaling of mTORC1. Indeed, several monogenic disorders resulting in overactive mTORC1 signaling, such as tuberous sclerosis complex, neurofibramatosis type 1, and PTEN (phosphatase and tensin homolog deleted on chromosome 10) harmatoma syndrome (Kwiat- 
kowski, 2003; Butler et al., 2005; Johannessen et al., 2005; Ehninger et al., 2008) have high comorbidity rates with autism spectrum disorders (ASDs), leading to the idea that altered protein synthesis in neurons may be a common phenotype central to the emergence of aberrant cognitive and behavioral characteristics related to ASD (Kelleher and Bear, 2008). Indeed, the well established role for mTORC1 in supporting protein synthesisdependent changes in postsynaptic function suggests that the resulting defects in postsynaptic adaptability stemming from dysregulated mTORC1 signaling are likely to contribute to these syndromes. Our findings reveal an integral role for dendritic mTORC1 in supporting retrograde modulation of presynaptic function, illustrating an alternative mode of synaptic regulation that may also be relevant for ASD and other disorders caused by synaptic dysfunction.

\section{References}

Aid T, Kazantseva A, Piirsoo M, Palm K, Timmusk T (2007) Mouse and rat BDNF gene structure and expression revisited. J Neurosci Res 85:525535. CrossRef Medline

An JJ, Gharami K, Liao GY, Woo NH, Lau AG, Vanevski F, Torre ER, Jones KR, Feng Y, Lu B, Xu B (2008) Distinct role of long 3' UTR BDNF mRNA in spine morphology and synaptic plasticity in hippocampal neurons. Cell 134:175-187. CrossRef Medline

Antion MD, Merhav M, Hoeffer CA, Reis G, Kozma SC, Thomas G, Schuman EM, Rosenblum K, Klann E (2008) Removal of S6K1 and S6K2 leads to divergent alterations in learning, memory, and synaptic plasticity. Learn Mem 15:29-38. CrossRef Medline

Aoto J, Nam CI, Poon MM, Ting P, Chen L (2008) Synaptic signaling by all-trans retinoic acid in homeostatic synaptic plasticity. Neuron 60:308320. CrossRef Medline

Baj G, Leone E, Chao MV, Tongiorgi E (2011) Spatial segregration of BDNF transcripts enables BDNF to differentially shape distinct dendritic compartments. Proc Natl Acad Sci U S A 108:16813-16818. CrossRef Medline

Ballou LM, Selinger ES, Choi JY, Drueckhammer DG, Lin RZ (2007) Inhibition of mammalian target of rapamycin signaling by 2-(morpholin-1yl)pyrimido[2,1-alpha]isoquinolin-4-one. J Biol Chem 282:24463-24470. CrossRef Medline

Bateup HS, Takasaki KT, Saulnier JL, Denefrio CL, Sabatini BL (2011) Loss of Tscl in vivo impairs hippocampal mGluR-LTD and increases excitatory synaptic function. J Neurosci 31:8862-8869. CrossRef Medline

Béique JC, Na Y, Kuhl D, Worley PF, Huganir RL (2011) Arc-dependent synapse-specific homeostatic plasticity. Proc Natl Acad Sci U S A 108: 816-821. CrossRef Medline

Blundell J, Kouser M, Powell CM (2008) Systemic inhibition of mammalian target of rapamycin inhibits fear memory reconsolidation. Neurobiol Learn Mem 90:28-35. CrossRef Medline

Branco T, Häusser M (2010) The single dendritic branch as a fundamental functional unit in the nervous system. Curr Opin Neurobiol 20:494-502. CrossRef Medline

Branco T, Staras K, Darcy KJ, Goda Y (2008) Local dendritic activity sets release probability at hippocampal synapses. Neuron 59:475-485. CrossRef Medline

Butler MG, Dasouki MJ, Zhou XP, Talebizadeh Z, Brown M, Takahashi TN, Miles JH, Wang CH, Stratton R, Pilarski R, Eng C (2005) Subset of individuals with autism spectrum disorders and extreme macrocephaly associated with germline PTEN tumour suppressor gene mutations. J Med Genet 42:318-321. CrossRef Medline

Cammalleri M, Lütjens R, Berton F, King AR, Simpson C, Francesconi W, Sanna PP (2003) Time-restricted role for dendritic activation of the mTOR-p70S6K pathway in the induction of late-phase long-term potentiation in the CA1. Proc Natl Acad Sci U S A 100:14368-14373. CrossRef Medline

Casadio A, Martin KC, Giustetto M, Zhu H, Chen M, Bartsch D, Bailey CH, Kandel ER (1999) A transient, neuron-wide form of CREB-mediated long-term facilitation can be stabilized at specific synapses by local protein synthesis. Cell 99:221-237. CrossRef Medline

Chiaruttini C, Sonego M, Baj G, Simonato M, Tongiorgi E (2008) BDNF mRNA splice variants display activity-dependent targeting to distinct hippocampal laminae. Mol Cell Neurosci 37:11-19. CrossRef Medline
Costa-Mattioli M, Sossin WS, Klann E, Sonenberg N (2009) Translational control of long-lasting synaptic plasticity and memory. Neuron 61:10-26. CrossRef Medline

Dash PK, Orsi SA, Moore AN (2006) Spatial memory formation and memory-enhancing effect of glucose involves activation of the tuberous sclerosis complex-mammalian target of rapamycin pathway. J Neurosci 26:8048-8056. CrossRef Medline

Ehninger D, Han S, Shilyansky C, Zhou Y, Li W, Kwiatkowski DJ, Ramesh V, Silva AJ (2008) Reversal of learning deficits in a Tsc2 + / - mouse model of tuberous sclerosis. Nat Med 14:843-848. CrossRef Medline

Frank CA, Kennedy MJ, Goold CP, Marek KW, Davis GW (2006) Mechanisms underlying the rapid induction and sustained expression of synaptic homeostasis. Neuron 52:663-677. CrossRef Medline

Gkogkas C, Sonenberg N, Costa-Mattioli M (2010) Translational control mechanisms in long-lasting synaptic plasticity and memory. J Biol Chem 285:31913-31917. CrossRef Medline

Gong B, Wang H, Gu S, Heximer SP, Zhuo M (2007) Genetic evidence for the requirement of adenylyl cyclase 1 in synaptic scaling of forebrain cortical neurons. Eur J Neurosci 26:275-288. CrossRef Medline

Gong R, Park CS, Abbassi NR, Tang SJ (2006) Roles of glutamate receptors and the mammalian target of rapamycin (mTOR) signaling pathway in activity-dependent dendritic protein synthesis in hippocampal neurons. J Biol Chem 281:18802-18815. CrossRef Medline

Goold CP, Nicoll RA (2010) Single-cell optogenetic excitation drives homeostatic synaptic depression. Neuron 68:512-528. CrossRef Medline

Groth RD, Lindskog M, Thiagarajan TC, Li L, Tsien RW (2011) \{beta\} $\mathrm{Ca} 2+/ \mathrm{CaM}$-dependent kinase type II triggers upregulation of GluA1 to coordinate adaptation to synaptic inactivity in hippocampal neurons. Proc Natl Acad Sci U S A 108:828-833. CrossRef Medline

Hessler NA, Shirke AM, Malinow R (1993) The probability of transmitter release at a mammalian central synapse. Nature 366:569-572. CrossRef Medline

Hoeffer CA, Klann E (2010) mTOR signaling: at the crossroads of plasticity, memory and disease. Trends Neurosci 33:67-75. CrossRef Medline

Hoeffer CA, Tang W, Wong H, Santillan A, Patterson RJ, Martinez LA, Tejada-Simon MV, Paylor R, Hamilton SL, Klann E (2008) Removal of FKBP12 enhances mTOR-Raptor interactions, LTP, memory, and perseverative/repetitive behavior. Neuron 60:832-845. CrossRef Medline

Hou L, Klann E (2004) Activation of the phosphoinositide 3-kinase-Aktmammalian target of rapamycin signaling pathway is required for metabotropic glutamate receptor-dependent long-term depression. J Neurosci 24:6352-6361. CrossRef Medline

Hou Q, Gilbert J, Man HY (2011) Homeostatic regulation of AMPA receptor trafficking and degradation by light-controlled single-synaptic activation. Neuron 72:806-818. CrossRef Medline

Huber KM, Kayser MS, Bear MF (2000) Role for rapid dendritic protein synthesis in hippocampal mGluR-dependent long-term depression. Science 288:1254-1257. CrossRef Medline

Ibata K, Sun Q, Turrigiano GG (2008) Rapid synaptic scaling induced by changes in postsynaptic firing. Neuron 57:819-826. CrossRef Medline

Inoki K, Li Y, Zhu T, Wu J, Guan KL (2002) TSC2 is phosphorylated and inhibited by Akt and suppresses mTOR signalling. Nat Cell Biol 4:648657. CrossRef Medline

Inoki K, Zhu T, Guan KL (2003) TSC2 mediates cellular energy response to control cell growth and survival. Cell 115:577-590. CrossRef Medline

Jakawich SK, Nasser HB, Strong MJ, McCartney AJ, Perez AS, Rakesh N, Carruthers CJ, Sutton MA (2010) Local presynaptic activity gates homeostatic changes in presynaptic function driven by dendritic BDNF synthesis. Neuron 68:1143-1158. CrossRef Medline

Jiang H, Vogt PK (2008) Constitutively active Rheb induces oncogenic transformation. Oncogene 27:5729-5740. CrossRef Medline

Johannessen CM, Reczek EE, James MF, Brems H, Legius E, Cichowski K (2005) The NF1 tumor suppressor critically regulates TSC2 and mTOR. Proc Natl Acad Sci U S A 102:8573-8578. CrossRef Medline

Kang H, Schuman EM (1996) A requirement for local protein synthesis in neurotrophin-induced hippocampal synaptic plasticity. Science 273: 1402-1406. CrossRef Medline

Kelleher RJ 3rd, Bear MF (2008) The autistic neuron: troubled translation? Cell 135:401-406. CrossRef Medline

Kim SH, Ryan TA (2009) Synaptic vesicle recycling at CNS snapses without AP-2. J Neurosci 29:3865-3874. CrossRef Medline 
Kwiatkowski DJ (2003) Tuberous sclerosis: from tubers to mTOR. Ann Hum Genet 67:87-96. CrossRef Medline

Lee MC, Yasuda R, Ehlers MD (2010) Metaplasticity at single glutamatergic synapses. Neuron 66:859-870. CrossRef Medline

Li N, Lee B, Liu RJ, Banasr M, Dwyer JM, Iwata M, Li XY, Aghajanian G, Duman RS (2010) mTOR-dependent synapse formation underlies the rapid antidepressant effects of NMDA antagonists. Science 329:959-964. CrossRef Medline

Liao GY, An JJ, Gharami K, Waterhouse EG, Vanevski F, Jones KR, Xu B (2012) Dendritically targeted Bdnf mRNA is essential for energy balance and response to leptin. Nat Med 18:564-571. CrossRef Medline

Lindskog M, Li L, Groth RD, Poburko D, Thiagarajan TC, Han X, Tsien RW (2010) Postsynaptic GluAl enables acute retrograde enhancement of presynaptic function to coordinate adaptation to synaptic inactivity. Proc Natl Acad Sci U S A 107:21806-21811. CrossRef Medline

Long X, Lin Y, Ortiz-Vega S, Yonezawa K, Avruch J (2005) Rheb binds and regulates the mTOR kinase. Curr Biol 15:702-713. CrossRef Medline

Ma XM, Blenis J (2009) Molecular mechanisms of mTOR-mediated translational control. Nat Rev Mol Cell Biol 10:307-318. CrossRef Medline

Maghsoodi B, Poon MM, Nam CI, Aoto J, Ting P, Chen L (2008) Retinoic acid regulates RARalpha-mediated control of translation in dendritic RNA granules during homeostatic synaptic plasticity. Proc Natl Acad Sci U S A 105:16015-16020. CrossRef Medline

Makino H, Malinow R (2009) AMPA receptor incorporation into synapses during LTP: the role of lateral movement and exocytosis. Neuron 64:381390. CrossRef Medline

Martin KC, Casadio A, Zhu H, Yaping E, Rose JC, Chen M, Bailey CH, Kandel ER (1997) Synapse-specific, long-term facilitation of aplysia sensory to motor synapses: a function for local protein synthesis in memory storage. Cell 91:927-938. CrossRef Medline

Murthy VN, Schikorski T, Stevens CF, Zhu Y (2001) Inactivity produces increases in neurotransmitter release and synapse size. Neuron 32:673682. CrossRef Medline

Neasta J, Ben Hamida S, Yowell Q, Carnicella S, Ron D (2010) Role for mammalian target of rapamycin complex 1 signaling in neuroadaptations underlying alcohol-related disorders. Proc Natl Acad Sci U S A 107: 20093-20098. CrossRef Medline

Nosyreva E, Kavalali ET (2010) Activity-dependent augmentation of spontaneous neurotransmission during endoplasmic reticulum stress. J Neurosci 30:7358-7368. CrossRef Medline

Onda H, Crino PB, Zhang H, Murphey RD, Rastelli L, Gould Rothberg BE, Kwiatkowski DJ (2002) Tsc2 null murine neuroepithelial cells are a model for human tuber giant cells, and show activation of an mTOR pathway. Mol Cell Neurosci 21:561-574. CrossRef Medline

Pattabiraman PP, Tropea D, Chiaruttini C, Tongiorgi E, Cattaneo A, Domenici L (2005) Neuronal activity regulates the developmental expression and subcellular localization of cortical BDNF mRNA isoforms in vivo. Mol Cell Neurosci 28:556-570. CrossRef Medline

Penney J, Tsurudome K, Liao EH, Elazzouzi F, Livingstone M, Gonzalez M, Sonenberg N, Haghighi AP (2012) TOR is required for the retrograde regulation of synaptic homeostasis at the Drosophila neuromuscular junction. Neuron 74:166-178. CrossRef Medline

Rabinowitch I, Segev I (2008) Two opposing plasticity mechanisms pulling a single synapse. Trends Neurosci 31:377-383. CrossRef Medline

Regehr WG, Carey MR, Best AR (2009) Activity-dependent regulation of synapses by retrograde messengers. Neuron 63:154-170. CrossRef Medline
Rosenmund C, Clements JD, Westbrook GL (1993) Non-uniform probability of glutamate release at a hippocampal synapse. Science 262:754757. CrossRef Medline

Schratt GM, Nigh EA, Chen WG, Hu L, Greenberg ME (2004) BDNF regulates the translation of a select group of mRNAs by a mammalian target of rapamycin-phosphatidylinositol 3-kinase-dependent pathway during neuronal development. J Neurosci 24:7366-7377. CrossRef Medline

Sharma A, Hoeffer CA, Takayasu Y, Miyawaki T, McBride SM, Klann E, Zukin RS (2010) Dysregulation of mTOR signaling in fragile X syndrome. J Neurosci 30:694-702. CrossRef Medline

Slipczuk L, Bekinschtein P, Katche C, Cammarota M, Izquierdo I, Medina JH (2009) BDNF activates mTOR to regulate GluR1 expression required for memory formation. PLoS One 4:e6007. CrossRef Medline

Soden ME, Chen L (2010) Fragile X protein FMRP is required for homeostatic plasticity and regulation of synaptic strength by retinoic acid. J Neurosci 30:16910-16921. CrossRef Medline

Steward O, Levy WB (1982) Preferential localization of polyribosomes under the base of dendritic spines in granule cells of the dentate gyrus. J Neurosci 2:284-291. Medline

Sutton MA, Ito HT, Cressy P, KempfC, Woo JC, Schuman EM (2006) Miniature neurotransmission stabilizes synaptic function via tonic suppression of local dendritic protein synthesis. Cell 125:785-799. CrossRef Medline

Sutton MA, Taylor AM, Ito HT, Pham A, Schuman EM (2007) Postsynaptic decoding of neural activity: eEF2 as a biochemical sensor coupling miniature synaptic transmission to local protein synthesis. Neuron 55:648 661. CrossRef Medline

Tang SJ, Reis G, Kang H, Gingras AC, Sonenberg N, Schuman EM (2002) A rapamycin-sensitive signaling pathway contributes to long-term synaptic plasticity in the hippocampus. Proc Natl Acad Sci U S A 99:467-472. CrossRef Medline

Tavazoie SF, Alvarez VA, Ridenour DA, Kwiatkowski DJ, Sabatini BL (2005) Regulation of neuronal morphology and function by the tumor suppressors Tsc1 and Tsc2. Nat Neurosci 8:1727-1734. CrossRef Medline

Tee AR, Manning BD, Roux PP, Cantley LC, Blenis J (2003) Tuberous sclerosis complex gene products, Tuberin and Hamartin, control mTOR signaling by acting as a GTPase-activating protein complex toward Rheb. Curr Biol 13:1259-1268. CrossRef Medline

Thiagarajan TC, Lindskog M, Tsien RW (2005) Adaptation to synaptic inactivity in hippocampal neurons. Neuron 47:725-737. CrossRef Medline

Tischmeyer W, Schicknick H, Kraus M, Seidenbecher CI, Staak S, Scheich H, Gundelfinger ED (2003) Rapamycin-sensitive signalling in long-term consolidation of auditory cortex-dependent memory. Eur J Neurosci 18: 942-950. CrossRef Medline

Voglmaier SM, Kam K, Yang H, Fortin DL, Hua Z, Nicoll RA, Edwards RH. (2006) Distinct endocytic pathways control the rate and extent of synaptic vesicle protein recycling. Neuron 51:71-84. CrossRef Medline

Wang CC, Held RG, Chang SC, Yang L, Delpire E, Ghosh A, Hall BJ (2011) A critical role for GluN2B-containing NMDA receptors in cortical development and function. Neuron 72:789-805. CrossRef Medline

Wang X, Proud CG (2006) The mTOR pathway in the control of protein synthesis. Physiology (Bethesda) 21:362-369. CrossRef Medline

Yang Q, Guan KL (2007) Expanding mTOR signaling. Cell Res 17:666-681. CrossRef Medline

Zoncu R, Efeyan A, Sabatini DM (2011) mTOR: from growth signal integration to cancer, diabetes and ageing. Nat Rev Mol Cell Biol 12:21-35. CrossRef Medline 